\title{
A unified framework for polycrystal plasticity with grain boundary evolution
}

\author{
Nikhil Chandra Admal ${ }^{\mathrm{a}, *}$, Giacomo Po ${ }^{\mathrm{b}}$, Jaime Marian ${ }^{\mathrm{a}, \mathrm{b}}$ \\ ${ }^{a}$ Materials Science and Engineering, University of California Los Angeles \\ ${ }^{b}$ Mechanical Engineering, University of California Los Angeles
}

\begin{abstract}
Plastic deformation in polycrystals is governed by the interplay between intra-granular slip and grain boundarymediated plasticity. However, while the role played by bulk dislocations is relatively well-understood, the contribution of grain boundaries (GBs) has only recently begun to be studied. GB plasticity is known to play a key role along with bulk plasticity under a wide range of conditions, such as dynamic recovery, superplasticity, severe plastic deformation, etc., and developing models capable of simultaneously capturing GB and bulk plasticity has become a topic of high relevance. In this paper we develop a thermodynamically-consistent polycrystal plasticity model capable of simulating a variety of grain boundary-mediated plastic processes in conjunction with bulk dislocation slip. The model starts from the description of a single crystal and creates lattice strain-free polycrystalline configurations by using a specially-designed multiplicative decomposition developed by the authors. This leads to the introduction of a particular class of geometrically necessary dislocations (GND) that define fundamental GB features such as misorientation and inclination. The evolution of the system is based on an energy functional that uses a non-standard function of the GND tensor to account for the grain boundary energy, as well as for the standard elastic energy. Our implementation builds on smooth descriptions of GBs inspired on diffuse-interface models of grain evolution for numerical convenience. We demonstrate the generality and potential of the methodology by simulating a wide variety of phenomena such as shear-induced GB sliding, coupled GB motion, curvature-induced grain rotation and shrinkage, and polygonization via dislocation sub-grain formation.
\end{abstract}

Keywords: A. grain boundary plasticity, microstructures, B. crystal plasticity, constitutive behavior, polycrystalline materials

\section{Introduction}

Engineering materials, particularly metallic alloys, almost always involve polycrystals, characterized by a conglomerate of grains with different crystal orientations ${ }^{1}$ separated by grain boundaries (GBs). Deformation models of polycrystalline materials must then necessarily account for grain boundary plasticity, such as that produced by shearinduced grain boundary motion, grain rotation and shrinkage, in addition to bulk or intra-grain plasticity. In fact, both classes of mechanisms often co-occur, such that it is typically quite difficult to distinguish between these in the general context of deformation. Never is this more true than in the well-known cases of recovery, grain growth, and recrystallization [1,2], which are commonplace processes taking place during high-stress deformation of metallic alloys -particularly at elevated temperature-, and where grain boundaries undergo microstructural transformations contemporaneously with bulk dislocations [3, 4]. Traditionally, however, both types of transformations have been modeled separately, as independent processes that are then linked via some phenomenological coupling (some of these will be discussed below). This has proven unsatisfactory to capture the full complexity observed during microstructural evolution at high stress and/or temperature. It thus urges to take a fresh look at the current theories to explore new avenues to model polycrystal plasticity with co-occurring grain boundary evolution.

The advent of highly-accurate and efficient atomistic methods has enabled the direct simulation of the mechanisms behind grain boundary evolution. Recent applications of molecular dynamics (MD) simulations has opened a new

\footnotetext{
${ }^{*}$ Corresponding author

Email address: admal002@g.ucla.com (Nikhil Chandra Admal)

${ }^{1}$ With respect to a global laboratory frame of reference.
} 
window into the rich physics and complexities associated with grain boundary phenomena $[5,6,7,8,9]$. In addition, other methods such as the phase field crystal (PFC) model [10,11], a continuum model which operates at atomic length and diffusive time scales, has been successfully used to simulate various mechanisms observed in grain boundary plasticity [12]. As well, discrete disclinations, a method pioneered by Taupin et al. [13], has been used successfully to obtain the energetics of grain boundaries, although the kinetics is still an open problem.

Evidently, the spatio-temporal limitations of atomistic, PFC, and disclination-based models preclude us from studying recovery and recrystallization on relevant timescales. Yet, new models can and should benefit from the understanding gained over the last few years from atomic-level simulations of grain boundaries structure and properties. Another outstanding limitation of most mesoscale continuum models is that they either cover the evolution of microstructure or deformation, but not both, lacking the generality to model annealing and recovery phenomena. For example, phase field models such as the Kobayashi-Warren-Carter (KWC) model [14, 15], or the multiphase field model [16, 17], and cellular automata [18] have been specifically devised to study the kinetics of grain growth, but do not capture deformation. On the other end of the spectrum are various crystal plasticity (CP) models for polycrystals which have been successfully applied to fundamental problems in materials deformation with a high rate of accuracy $[19,20,21]$. These models are geared towards modeling bulk plasticity, with fixed grain boundaries playing a surrogate role of describing the variations in slip planes and elastic moduli, and at times providing the necessary back stress to resist the build up of bulk dislocations [22, 23], or to incorporate grain boundary sliding [24, 25]. A notable exception to the above observation is a class of sharp-interface models developed by Cahn et al. [5], Basak and Gupta [26], Frolov and Mishin [27], wherein grain boundaries move and result in macroscopic deformation. Nevertheless, since these models are not rooted in crystal plasticity they are less equipped to deal with the interaction of bulk dislocations and grain boundaries, which plays a key role during the stages of recovery and recrystallization.

Very interesting advances have been proposed recently by coupling phase field/level set/cellular automata models to standard crystal plasticity $[28,29,30,31,32,33]$ to include microstructure evolution along with deformation. In these coupled models, the plastic strain or the stress are updated using CP and the resulting dislocation density is passed to the grain boundary evolution model, which uses it in a penalty function to steer grain boundary evolution to areas of high accumulated plastic strain. Therefore, within this framework, deformation is a result of only bulk crystal plasticity, and grain boundary motion does not contribute to macroscopic deformation. This implies phenomena such as shear-induced grain boundary motion, grain sliding and subgrain nucleation are beyond the reach of such models.

While the power of these coupled formulations to simulate grain boundary kinetics along with deformation at different levels of accuracy must be recognized, in this paper we propose a model that can simulate bulk and grain boundary plasticity in unison. The model stems from a unifying framework developed recently by the authors [34]. Unlike in bulk polycrystal plasticity, where the reference configuration (represented by the vector $\boldsymbol{X}$ ) is a strain-free polycrystal (resulting in piecewise-constant slip systems and elastic moduli) with initial state described as $\boldsymbol{F}^{\mathrm{L}}=$ $\boldsymbol{F}^{\mathrm{P}}=\boldsymbol{I}$ (where $\boldsymbol{F}^{\mathrm{L}}$ and $\boldsymbol{F}^{\mathrm{P}}$ are the elastic and plastic part of the deformation gradient $\boldsymbol{F}$, and $\boldsymbol{I}$ is the identity tensor), in this framework we begin with a strain-free single crystal as the reference configuration, and grain boundaries emerge naturally as arrangements of geometrically necessary dislocations (GNDs) that preserve the compatibility of the deformation. This is done using a special decomposition of $\boldsymbol{F}(\boldsymbol{X}, 0) \equiv \boldsymbol{I}$ by having $\boldsymbol{F}^{\mathrm{P}}(\boldsymbol{X}, 0)$ as a smoothened piecewise-constant rotation field and $\boldsymbol{F}^{\mathrm{L}}=\boldsymbol{F}^{\mathrm{pT}}$. The above construction results in a lattice strain-free diffuseinterface polycrystal as the initial state, with grain misorientations arising due to the presence of GNDs. The unique feature of this framework is that grain boundaries are not viewed as a new class of defects, but an integral part of the microstructure, thus making the interaction of bulk dislocation with grain boundaries a tractable problem.

This notion of grain boundary GNDs is subsequently used to construct a system free energy expressed as a nonstandard function of the GND density along with the classical elastic energy. This free energy is then used in a dissipative thermodynamic framework that leads to evolution equations that can be integrated under a variety of conditions representative of elementary grain boundary processes. We demonstrate the potential of this unified framework and the constitutive law by simulating coupled grain boundary motion, grain sliding, rotation and subgrain nucleation during dynamic recovery.

The paper is organized as follows: after this Introduction section we discuss the phase field approach by Kobayashi et al. $[15,14]$ in Section 2, which we use a starting point to elaborate our theory. We then introduce the kinematic framework to include grain boundary kinetics in Section 3, followed by the derivation of balance laws in Sections 4 and 5. In Section 6, we develop a thermodynamically-consistent constitutive law, followed by results in Section 7, where we discuss the numerical aspects of the model and show how the model handles the various grain boundary processes mentioned above, including polygonization. We finalize with a discussion and the conclusions. We use 
standard notation throughout the paper unless noted otherwise. ${ }^{2}$

\section{The Kobayashi-Warren-Carter model for crystal grain evolution}

The Kobayashi-Warren-Carter (KWC) model, proposed by Kobayashi et al. [15, 14] is a phase-field model to study grain evolution in polycrystalline materials. The model for a two-dimensional polycrystal $\Omega_{0} \in \mathbb{R}^{2}$, consists of two scalar order parameters $\phi$ and $\theta$, representing, respectively, phase state and crystal orientation. $\phi$ ranges between 0 (disordered phase) and 1 (crystalline state), while $\theta$ represents the orientation ${ }^{3}$ of a crystal. The KWC free energy functional $\mathcal{W}^{\mathrm{KWC}}$ is given by

$$
\mathcal{W}^{\mathrm{KWC}}[\phi, \theta]=\int_{\Omega_{0}} \psi^{\mathrm{KWC}} d V
$$

with

$$
\psi^{\mathrm{KWC}}(\phi, \nabla \phi, \nabla \theta)=\frac{\alpha^{2}}{2}|\nabla \phi|^{2}+f(\phi)+g(\phi) s|\nabla \theta|+\frac{\epsilon^{2}}{2}|\nabla \theta|^{2},
$$

and

$$
\begin{aligned}
& f(\phi)=e(\phi-1)^{2}, \\
& g(\phi)=\phi^{2} .
\end{aligned}
$$

The constants $e, \alpha, \epsilon$ and $s$ are material constants. The Euler-Lagrange equations resulting from taking independent variations of $\psi^{\mathrm{KWC}}$ with respect to $\phi$ and $\theta$ are given by

$$
\begin{aligned}
b^{\phi} \dot{\phi} & =\alpha^{2} \triangle \phi-f_{\phi}-g_{\phi} s|\nabla \theta|, \\
b^{\theta} \dot{\theta} & =\operatorname{Div}\left[\epsilon^{2} \nabla \theta+g s \frac{\nabla \theta}{|\nabla \theta|}\right],
\end{aligned}
$$

where $b^{\phi}$ and $b^{\theta}$ are the inverse mobilities of the phase fields $\phi(\boldsymbol{X})$ and $\theta(\boldsymbol{X})$, respectively. Lobkovsky and Warren [35] have shown that the sharp-interface limit of equations (3a) and (3b) gives rise to grain rotation and grain boundary motion by curvature.

The term $\nabla \theta /|\nabla \theta|$ makes (3b) a singular diffusive equation ${ }^{4}$. This is a consequence of having the non-standard weighted total variation term $g(\phi) s|\nabla \theta|$ in (2). This term tends to localize the grain boundary, while $\epsilon^{2}|\nabla \theta|^{2} / 2$ tends to diffuse it. The opposing nature of the two terms can be easily examined by studying the steady state solution of a bicrystal (see Kobayashi and Giga [36]). If $\epsilon=0$ and $s \neq 0$, then the steady-state solution for $\theta(\boldsymbol{X})$ is a step function, resulting in a sharp-interface bicrystal. Conversely, for $s=0$ and $\epsilon \neq 0, \theta$ is a linear function in steady state. The two terms in $|\nabla \theta|$ therefore act together giving rise to grain boundaries with finite width. In addition to grain boundary regularization, $\epsilon$ plays an important role in the mobility of the grain boundary, as will be shown below.

For the sake of numerical convenience, Kobayashi and Giga [36] proposed to replace the singular term in (3b), $|\nabla \theta|$, with the non-singular approximation:

$$
p(|\nabla \theta|):=\frac{\ln (\cosh (\gamma|\nabla \theta|))}{\gamma}
$$

where $\gamma$ is an adjustable parameter. This term converges to $|\nabla \theta|$ in the limit $\gamma \rightarrow \infty$. The resulting Euler-Lagrange equations are now given by

$$
\begin{aligned}
b^{\phi} \dot{\phi} & =\alpha^{2} \triangle \phi-f,,_{\phi}-g,,_{\phi} s p, \\
b^{\theta} \dot{\theta} & =\operatorname{Div}\left[\epsilon^{2} \nabla \theta+g s p,|\nabla \theta|\right] .
\end{aligned}
$$

\footnotetext{
${ }^{2}$ All scalar, tensor and vector fields in this paper are assumed to be infinitely smooth unless otherwise stated. Bold letters are used to represent vectors and tensors. The derivative of a field $f(\bullet)$ with respect to its argument $\bullet$ is denoted by $f, \bullet$. $\nabla$ denotes the gradient operator, and its action on vector and scalar fields in indicial notation is defined as $[\nabla \boldsymbol{v}]_{i j}:=v_{i, j}$ and $[\nabla a]_{i}:=a,,_{i}$ respectively. The divergence operator is denoted by Div, and its action on vector and tensor fields in indicial notation (with Einstein summation convention) is defined as Div $\boldsymbol{v}:=v_{i, i}$ and $[\operatorname{Div} \boldsymbol{T}]_{i}:=T_{i j, j}$ respectively. The volume and surface elements in the reference configuration are denoted by $d V$ and $d A$ respectively.

${ }^{3}$ Since the system is two-dimensional, a scalar is sufficient to represent the orientation of a crystal.

${ }^{4}$ See [36] for an excellent introduction to singular diffusive equations
} 


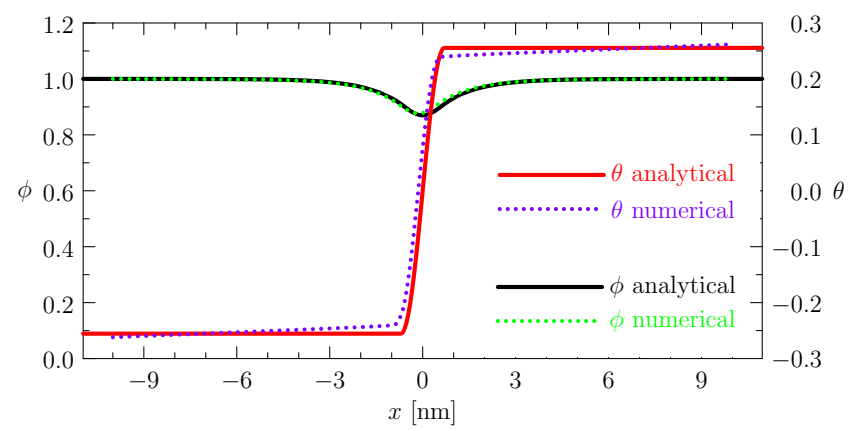

Figure 1: Comparison of the steady state numerical solution to the steady state analytical solution given in Appendix A.
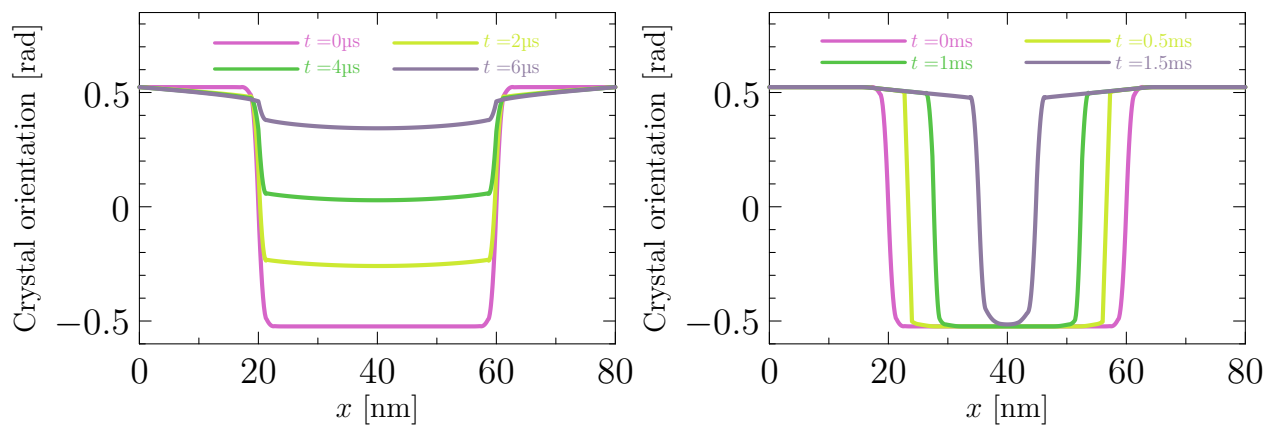

Figure 2: Evolution of the $\theta$ field during rotation and shrinkage of a circular grain according to the KWC model.

For a nominal parameter set, the above model results in the steady state profiles given in Fig. 1a for $\phi$ and $\theta$. As the figures show, within each grain $\phi=1$, which symbolizes perfect crystalline order. $\phi$ drops in value at the grain boundary. For its part, $\theta$ behaves as a regularized step function, with the step height representing the misorientation. An important aspect of the KWC model is that the evolution of both phase field variables is linked due to the cross-term in (3a), which attaches the grain boundary to the evolution of both variables. The numerical details behind these profiles as well as details about the solution procedure can be found in Appendix A.

One of the most attractive features of the KWC model is that its free energy functional allows for processes such as grain rotation and shrinkage. The two modes can be explored independently by appropriately choosing the mobilities in eqs. $3 \mathrm{a}$ and $3 \mathrm{~b}$. For example, for a circular grain of radius $20 \mathrm{~nm}$ with a misorientation of $60^{\circ}$ embedded inside a square domain, the two processes are simulated in Fig. 2, with rotation manifesting itself as changes in misorientation -without grain boundary displacement-, and shrinkage as a constant misorientation with inward motion of the grain boundaries.

Despite this great flexibility, the KWC free energy functional is subjected to two fundamental limitations. The first is that it is completely phenomenological, without any connection to the underlying plastic mechanisms. In second place, because the driving force for grain boundary motion modeled using the KWC functional arises only due to its curvature, it is insensitive to external stress. Therefore, the KWC functional cannot model phenomena such as shearinduced grain boundary motion. The main aim of this paper is to devise a polycrystal plasticity model that can address these shortcomings to study the general response of polycrystalline systems to deformation.

\section{Kinematics of polycrystal plasticity}

The aim of this section is to present the kinematics behind a continuum polycrystal plasticity model capable of simultaneously modeling bulk deformation and grain boundary evolution. First, in Section 3.1, we define the kinematic variables of bulk polycrystal plasticity with fixed grain boundaries and identify the challenges involved in their generalization to include grain boundary evolution. In Section 3.2, we discuss the central idea of the paper in order to address the challenges of bulk polycrystal plasticity by introducing an abstract kinematic framework of constructing grain boundaries using geometrically necessary dislocations. Using the kinematic framework introduced in Section 3.2, 
a thermodynamically-consistent polycrystal plasticity model with grain boundary energy is developed in Sections 4, 5 and 6.

\subsection{Kinematics of bulk polycrystal plasticity}

As is customary in continuum mechanics, a body is represented as an open subset $\mathcal{B}$ of the three-dimensional Euclidean space $\mathbb{R}^{3}$. The deformation of the body is described relative to a reference configuration $\mathcal{B}^{0} \in \mathbb{R}^{3}$. A point in $\mathcal{B}^{0}$ is referred to as a material point, and it is denoted by $\boldsymbol{X}$. A time-dependent deformation of the body is given using a one-to-one deformation map $\boldsymbol{y}(\boldsymbol{X}, t)$ such that $\operatorname{det} \boldsymbol{F} \neq 0$, where

$$
\boldsymbol{F}(\boldsymbol{X}, t):=\nabla \boldsymbol{y}
$$

where $\nabla$ is the gradient with respect to the material coordinate. A central idea in the theory of crystal plasticity is the multiplicative decomposition of the material deformation gradient into lattice and plastic components [37, 38, 39], i.e.

$$
\boldsymbol{F}=\boldsymbol{F}^{\mathrm{L}} \boldsymbol{F}^{\mathrm{P}},
$$

where $\boldsymbol{F}^{\mathrm{L}}$ denotes the lattice distortion, and $\boldsymbol{F}^{\mathrm{P}}$ denotes the lattice-invariant plastic distortion. In this paper, $\boldsymbol{F}^{\mathrm{P}}$ represents a lattice-invariant plastic shear associated to the mechanism of dislocation slip. Note that unlike $\boldsymbol{F}, \boldsymbol{F}^{\mathrm{L}}$ and $\boldsymbol{F}^{\mathrm{P}}$ are $n o t$ necessarily gradients of vector fields. $\boldsymbol{F}^{\mathrm{P}}$ maps the infinitesimally small material element $d \boldsymbol{X}$ to $\boldsymbol{F}^{\mathrm{P}} d \boldsymbol{X}$. The collection of distorted material elements $\boldsymbol{F}^{\mathrm{P}} d \boldsymbol{X}$ is denoted by lattice configuration. Therefore, $\boldsymbol{F}^{\mathrm{P}}$ maps the reference configuration to lattice configuration, and $\boldsymbol{F}^{\mathrm{L}}$ maps the lattice configuration to the deformed configuration.

Since $\boldsymbol{F}^{\mathrm{P}}$ represents plastic distortion due to dislocation slip, its evolution is given using the available families of dislocation slip planes $s^{\alpha}$ and their normal $\boldsymbol{m}^{\alpha,}$, along with the slip rates $v^{\alpha} \cdot \boldsymbol{F}^{\mathrm{P}}$ is evolved using the flow rule and the initial condition

$$
\begin{aligned}
\dot{\boldsymbol{F}}^{\mathrm{P}} & =\boldsymbol{L}^{\mathrm{P}} \boldsymbol{F}^{\mathrm{P}}, \\
\boldsymbol{F}^{\mathrm{P}}(\boldsymbol{X}, 0) & =\boldsymbol{I}, \quad \boldsymbol{X} \in \mathcal{B}^{0}
\end{aligned}
$$

respectively, where $\boldsymbol{L}^{\mathrm{P}}$ is commonly referred to as the plastic velocity gradient. In single crystal plasticity, $\boldsymbol{L}^{\mathrm{P}}$ is defined as the linear combination of slip contributions on all available slip systems, denoted by superindex $\alpha$, where each slip system is defined by a unique set of $s^{\alpha}$ and $\boldsymbol{m}^{\alpha}$ :

$$
\boldsymbol{L}^{\mathrm{P}}:=\sum_{\alpha=1}^{A} v^{\alpha} \boldsymbol{s}^{\alpha} \otimes \boldsymbol{m}^{\alpha} .
$$

The Schmid tensor $\boldsymbol{s}^{\alpha} \otimes \boldsymbol{m}^{\alpha}$ projects the amount of slip on each system w.r.t. the laboratory frame of reference. For bulk polycrystal plasticity with fixed grain boundaries, $s^{\alpha}$ and $\boldsymbol{m}^{\alpha}$ in (9) are replaced by $\boldsymbol{R}^{0} \boldsymbol{s}^{\alpha}$ and $\boldsymbol{R}^{0} \boldsymbol{m}^{\alpha}$ respectively, where $\boldsymbol{R}^{0}$ is a fixed piecewise constant rotation tensor describing the relative orientation of each grain. ${ }^{6}$

In single crystal plasticity, the elastic free energy density, denoted by $\psi$, is assumed to be a function of the lattice Lagrangian strain

$$
\mathbb{E}^{\mathrm{L}}:=\left(\left(\boldsymbol{F}^{\mathrm{L}}\right)^{\mathrm{T}} \boldsymbol{F}^{\mathrm{L}}-\boldsymbol{I}\right) / 2,
$$

while in polycrystal plasticity, $\psi\left(\mathbb{E}^{\mathrm{L}}\right)$ is replaced by $\psi\left(\boldsymbol{R}^{0} \mathbb{E}^{\mathrm{L}} \boldsymbol{R}^{0 \mathrm{~T}}\right)$, reflecting the piecewise constant elastic response of each grain. Therefore, bulk polycrystal plasticity is equivalent to single crystal plasticity with save for the difference that the slip directions, slip plane normals, and the elastic moduli are piecewise constant in the former.

The primary aim of this paper is to generalize the above described bulk polycrystal plasticity model to include grain boundary evolution. We first note that an evolving grain boundary may not only affect bulk deformation but it also results in plastic distortion. For example, consider a circular grain embedded in a larger ambient grain, with a misorientation of $\Delta \theta$ which results in a nonzero grain boundary energy. One possible mechanism to decrease the internal energy is for the circular grain to shrink thus decreasing the grain boundary surface area. As the grain boundary

\footnotetext{
${ }^{5}$ The vectors $\boldsymbol{s}^{\alpha}$ and $\boldsymbol{m}^{\alpha}$ exist in the lattice configuration.

${ }^{6}$ In other words, the slip direction and the slip plane normals are piecewise constant in bulk polycrystal plasticity.
} 


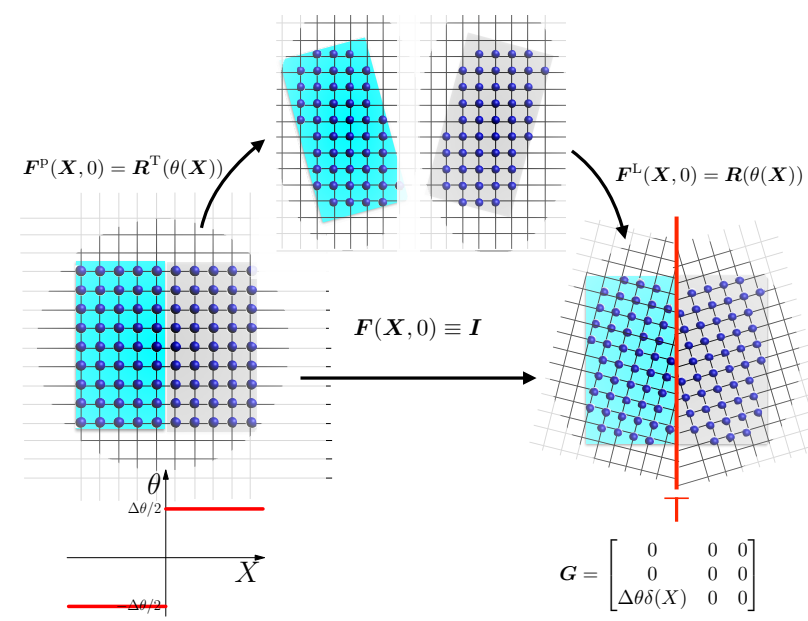

Figure 3: In two-dimensions, the above construction results in exactly two non-zero components $\left(G_{31}\right.$ and $\left.G_{32}\right)$ of $\boldsymbol{G}$. In particular, for a symmetric tilt boundary oriented as shown above, $G_{32} \equiv 0$ when $\theta$ is a step function.

sweeps through the material, the lattice in the swept region rotates by an angle of $\Delta \theta$, while the rest of the lattice remains unchanged. If $\boldsymbol{F}^{\mathrm{P}}$ is equal to identity during this process, then this results in an incompatible $\boldsymbol{F}$. This is a conclusive evidence that $\boldsymbol{F}^{\mathrm{P}} \not \equiv \boldsymbol{I}$ in the swept area. In other words, grain boundary motion always results in plastic distortion. Next, we present our approach for modeling grain boundary evolution within the framework of polycrystal plasticity.

\subsection{An abstract kinematic framework to include grain boundary plasticity}

The central idea behind our approach is to define grain boundaries as dislocation arrangements with their own unique properties and densities that suffice to specify the key properties of each boundary. One of the most important advantages of this idea is that plastic distortion due to grain boundary motion emerges naturally from the original flow rule given in (8a), without the need to specify extra mechanisms to account for their contributions to plastic deformation. In Section 7 we show that this approach can indeed model phenomena such as shear-induced grain boundary motion, grain boundary sliding and grain rotation.

We begin by noting that a polycrystalline material such as that discussed in Section 3.1 is modeled as a sharpinterface system with $\boldsymbol{R}^{0}(\boldsymbol{X}) \in S O(3)$, a step function in the space of special orthogonal tensor fields, representing the lattice rotation field in the polycrystal, with piecewise-constant values in each grain. In addition, recall that in Section 3.1 the reference configuration is a lattice strain-free polycrystal with the initial state:

$$
\boldsymbol{F}(\boldsymbol{X}, 0)=\boldsymbol{F}^{\mathrm{L}}(\boldsymbol{X}, 0)=\boldsymbol{F}^{\mathrm{P}}(\boldsymbol{X}, 0) \equiv \boldsymbol{I} .
$$

In the current framework, we start instead with a single crystal as the reference configuration, and an initial state given by:

$$
\boldsymbol{F}^{\mathrm{L}}(\boldsymbol{X}, 0)=\boldsymbol{R}^{0}(\boldsymbol{X}), \quad \boldsymbol{F}^{\mathrm{P}}(\boldsymbol{X}, 0)=\boldsymbol{R}^{0}(\boldsymbol{X})^{\mathrm{T}},
$$

resulting in

$$
\boldsymbol{F}(\boldsymbol{X}, 0) \equiv \boldsymbol{I}
$$

Fig. 3 shows the decomposition given in eqs. (12) and (13) for a single grain boundary in a bicrystal. Recall that $\boldsymbol{F}^{\mathrm{P}}$ deforms the material leaving the lattice fixed, while $\boldsymbol{F}^{\mathrm{L}}$ deforms the lattice resulting in a total deformation gradient $\boldsymbol{F}$ that is compatible. Comparing the reference and the final configurations in Fig. 3, this assertion seems contradictory, since the material is shown to be deformed even though $\boldsymbol{F} \equiv \boldsymbol{I}$. We have resolved this apparent contradiction resorting to the notion of weak-convergence discussed in our recent work [34], by which interpreting $\boldsymbol{F}^{\mathrm{P}}(\boldsymbol{X}, 0)=\boldsymbol{R}^{0 \mathrm{~T}}(\boldsymbol{X})$ and $\boldsymbol{F} \equiv \boldsymbol{I}$ for a discrete lattice in an average sense, as a sequence of lattice-invariant deformations, proves the correctness of eqs. (12) and (13). 
The piecewise-constant field $\boldsymbol{R}^{0}(\boldsymbol{X})$ results in a sharp-interface polycrystal with surfaces at which $\boldsymbol{R}^{0}(\boldsymbol{X})$ jumps signaling the existence of a grain boundaries. Alternatively, these jumps can be replaced with a smoothened version $\widetilde{\boldsymbol{R}}^{0}(\boldsymbol{X}) \in S O(3)$ of $\boldsymbol{R}^{0}$, resulting in a diffuse-interface grain boundary. ${ }^{7}$ The smoothening results in a more convenient numerical implementation (see Admal et al. [34]) of the kinematic variables, as they can be discretized using continuous finite elements. In addition to numerical simplicity, we will show in Section 6 that a diffuse-grain boundary description enables us to construct a grain boundary energy inspired from the KWC energy functional.

An important consequence of the decomposition given in eq. (12) is that the resulting sharp/diffuse-interface polycrystal is lattice strain-free since the Lagrangian strain, defined in (10), is equal to zero. Therefore, eq. (12) describes a polycrystalline state which is obtained from a reference single crystal by the right amount of slip in each grain such that grains undergo relative rotation but the underlying lattice in the polycrystal remains strain-free.

The main advantage of the initial kinematic state constructed using (12) is that we have immediate access to the grain boundary dislocation density content in the form of the geometrically necessary dislocation density $G$ tensor defined as

$$
\boldsymbol{G}=\boldsymbol{F}^{\mathrm{P}} \operatorname{Curl} \boldsymbol{F}^{\mathrm{P}}
$$

where Curl denotes the $\operatorname{curl}^{8}$ of a tensor field with respect to the material/reference coordinate. See Acharya [40] for a discussion on the physical significance of the above definition, and other alternatives. For a given normal $\boldsymbol{n}$ in the lattice configuration, the vector $G^{\mathrm{T}} \boldsymbol{n}$ measures the net Burgers vector of dislocation lines per unit area passing through a plane of normal $\boldsymbol{n}$, in the lattice configuration. From the definition of $\boldsymbol{G}$ in (14), it is clear that for a sharpinterface polycrystal, $G$ is not a function but a distribution whose support is the collection of grain boundary surfaces. For instance, the bicrystal constructed in Fig. 3 results in $G_{31}=\theta_{0} \delta(\boldsymbol{X})$, while all other components are identically equal to zero. On the other hand, $G_{32}$ is not equal to zero for a flat diffuse grain boundary. This can be seen using a smoothened step function $\widetilde{\theta}\left(X_{1}\right)$ describing the orientation of the two-dimensional bicrystal, and $\boldsymbol{F}^{\mathrm{P}}=\boldsymbol{R}^{0 \mathrm{~T}}\left(\widetilde{\theta}\left(X_{1}\right)\right)$. The GND tensor evaluated using (14) results in

$$
G_{31}=-\cos \left(\widetilde{\theta}\left(X_{1}\right)\right) \widetilde{\theta}^{\prime}\left(X_{1}\right), \quad G_{32}=-\sin \left(\widetilde{\theta}\left(X_{1}\right)\right) \widetilde{\theta}^{\prime}\left(X_{1}\right),
$$

and rest of the components of $G$ identically equal to zero. Constructing grain boundaries using GNDs has the advantage that the evolution of $G$ not only tracks bulk dislocations but also sharp/diffuse grain boundaries.

In Section 7.2, we show that constructing grain boundaries using GNDs enables us to simulate shear-induced grain boundary motion since dislocations move under applied shear stress. In addition, we show in Section 7.3 that grain boundary motion by curvature is also possible within this framework by including grain boundary energy in the free energy through a non-standard dependence on $G$ and a phase field variable $\phi$ which has the same significance in this model as in the KWC model. The exact functional dependence on $\boldsymbol{G}$ and $\phi$ will be made clear in Section 6 when we make the connection with the KWC energy functional.

Summarising the kinematics of the polycrystal plasticity model, we have the displacement $\boldsymbol{u}:=\boldsymbol{y}-\boldsymbol{X}$, slip rates $v^{\alpha}(\alpha=1, \ldots, A)$, and $\phi$ as the independent kinematic variables. Corresponding to these kinematic variables, we introduce conjugate forces, power and a virtual power formulation in the next section, and derive the necessary momentum balance laws.

\section{Virtual power formulation of the standard and microscopic force balance}

In this section, we develop a virtual power formulation for the kinematic variables introduced in Section 3 based on the framework developed by Gurtin [20]. Let $\mathcal{P}^{0} \subset \mathcal{B}^{0}$ denote an arbitrary part of the body. The formulation of the principle of virtual work is based on the balance between external power $\mathcal{W}\left(\mathcal{P}^{0}\right)$ expended on $\mathcal{P}^{0}$, and the internal power $\mathcal{I}\left(\mathcal{P}^{0}\right)$ expended within $\mathcal{P}^{0}$. We assume that the internal power is expended by a stress $\boldsymbol{P}$ conjugate of $\boldsymbol{F}$, a

\footnotetext{
${ }^{7}$ Note that the components of a $\widetilde{\boldsymbol{R}}^{0} \in S O(3)$ are not obtained by regularizing the components of its piecewise-constant counterpart $\boldsymbol{R}^{0}(\boldsymbol{X})$. Instead, it has to be constructed by appropriately regularizing the axis and angle descriptors of $\boldsymbol{R}^{0}(\boldsymbol{X})$.

${ }^{8}$ The curl of a tensor field $\boldsymbol{T}$ is defined as

$$
(\operatorname{Curl} T) \boldsymbol{n}:=\operatorname{Curl}\left(\boldsymbol{T}^{\mathrm{T}} \boldsymbol{n}\right),
$$

where $\boldsymbol{n}$ is an arbitrary constant vector, and the curl on the right-hand-side of the above equation is the curl of a vector field defined as $(\text { Curl } v)_{i}=$ $\epsilon_{i j k} v_{j, k}$, for any vector field $\boldsymbol{v}$. In indicial notation, it is given by $(\operatorname{Curl} \boldsymbol{T})_{i j}=\epsilon_{i p q} T_{j q, p}$.
} 
stress vector $\mathfrak{p}$ conjugate to $\nabla \phi$, a scalar internal microscopic force $\pi$ power-conjugate to $\dot{\phi}$, and for each slip system $\alpha$, a scalar internal microscopic force $\Pi^{\alpha}$ power-conjugate to the slip $v^{\alpha}$, and a vector microscopic stress $\boldsymbol{\xi}^{\alpha}$ power conjugate to $\nabla v^{\alpha}$. In other words, the internal power expended within $\mathcal{P}^{0}$ is given by

$$
\mathcal{I}\left(\mathcal{P}^{0}\right)=\int_{\mathcal{P}^{0}}(\boldsymbol{P} \cdot \dot{\boldsymbol{F}}+\mathfrak{p} \cdot \nabla \dot{\phi}+\pi \dot{\phi}) d V+\sum_{\alpha} \int_{\mathcal{P}^{0}}\left(\Pi^{\alpha} v^{\alpha}+\boldsymbol{\xi}^{\alpha} \cdot \nabla v^{\alpha}\right) d V .
$$

The external power on $\mathcal{P}^{0}$ is assumed to be a result of body forces, and various traction forces acting on $\partial \mathcal{P}^{0}$. The structure of the work done by external traction forces is obtained by examining the surface integrals resulting from the expression for internal power given in (16), i.e.

$$
\begin{aligned}
\mathcal{I}\left(\mathcal{P}^{0}\right)= & \int_{\partial \mathcal{P}^{0}}\left[\boldsymbol{P} \boldsymbol{N} \cdot \dot{\boldsymbol{y}}+\mathfrak{p} \cdot \boldsymbol{N} \dot{\phi}+\sum_{\alpha}\left(\boldsymbol{\xi}^{\alpha} \cdot \boldsymbol{N}\right) v^{\alpha}\right] d A+ \\
& \int_{\mathcal{P}^{0}}[\dot{\phi}(\pi-\operatorname{Div} \mathfrak{p})-\dot{\boldsymbol{y}} \cdot \operatorname{Div} \boldsymbol{P}] d V+\sum_{\alpha} \int_{\mathcal{P}^{0}}\left(\Pi^{\alpha}-\operatorname{Div} \boldsymbol{\xi}^{\alpha}\right) v^{\alpha} d V .
\end{aligned}
$$

Note that the surface integrals in (17) consist of a macroscopic surface traction that is conjugate to $\dot{\boldsymbol{y}}$, and two classes of microscopic tractions that are conjugate to $\dot{\phi}$ and slip rates respectively. This suggests the following form for the external power:

$$
\mathcal{W}\left(\mathcal{P}^{0}\right)=\int_{\partial \mathcal{P}^{0}}\left[\boldsymbol{t}(\boldsymbol{N}) \cdot \dot{\boldsymbol{y}}+s(\boldsymbol{N}) \dot{\phi}+\Xi^{\alpha}(\boldsymbol{N}) v^{\alpha}\right] d A
$$

where $\boldsymbol{t}$ is the macroscopic traction conjugate to $\dot{\boldsymbol{y}}$, and $s$ and $\Xi^{\alpha}$ are microscopic tractions conjugate to $\dot{\phi}$ and $v^{\alpha}$ respectively.

Taking independent variations in $\dot{\boldsymbol{y}}, \dot{\phi}$ and $v^{\alpha}(\alpha=1, \ldots, A)$, we arrive at the necessary macroscopic and microscopic force balance equations.

Macroscopic force balance

$$
\begin{aligned}
\operatorname{Div} \boldsymbol{P} & =\mathbf{0} \text { in } \mathcal{B}^{0}, \\
\boldsymbol{t} & =\boldsymbol{P} \boldsymbol{N} \text { on } \partial \mathcal{B}^{0} .
\end{aligned}
$$

Microscopic force balance for each slip system $\alpha=1, \ldots, A$

$$
\begin{aligned}
\operatorname{Div} \boldsymbol{\xi}^{\alpha}-\Pi^{\alpha} & =\mathbf{0} \text { in } \mathcal{B}^{0}, \\
\Xi^{\alpha} & =\boldsymbol{\xi}^{\alpha} \cdot \boldsymbol{N} \text { on } \partial \mathcal{B}^{0} .
\end{aligned}
$$

Microscopic force balance for $\phi$

$$
\begin{aligned}
\operatorname{Div} \mathfrak{p}-\pi & =\mathbf{0} \text { in } \mathcal{B}^{0}, \\
s & =\mathfrak{p} \cdot \boldsymbol{N} \text { on } \partial \mathcal{B}^{0} .
\end{aligned}
$$

\section{Energy Balance}

In this section, we introduce the law of the balance of energy, and the second law of thermodynamics expressed in the form of the Clausius-Duhem inequality. Table 1 list the notation for the various physical quantities introduced in this section. All densities defined in Table 1 are with respect to the reference volume.

Energy balance for an arbitrary subpart $\mathcal{P}^{0}$ of the body is given by

$$
\overline{\int_{\mathcal{P}^{0}} \epsilon d V}=-\int_{\partial \mathcal{P}^{0}} \boldsymbol{q} \cdot \boldsymbol{n} d A+\int_{\mathcal{P}^{0}} r d V+\mathcal{W}\left(\mathcal{P}^{0}\right),
$$

where $q$ denotes the heat flux vector, and $r$ denotes the external heat source. By the principle of virtual work, we have $\mathcal{W}\left(\mathcal{P}^{0}\right)=\mathcal{I}\left(\mathcal{P}^{0}\right)$. Therefore, substituting (16) into the above equation, we obtain the following in a differential form:

$$
\dot{\epsilon}=-\operatorname{Div} \boldsymbol{q}+r+(\boldsymbol{P} \cdot \dot{\boldsymbol{F}}+\mathfrak{p} \cdot \nabla \dot{\phi})+\sum_{\alpha}\left(\Pi^{\alpha} v^{\alpha}+\boldsymbol{\xi}^{\alpha} \cdot \nabla v^{\alpha}\right)+\pi \dot{\phi} .
$$




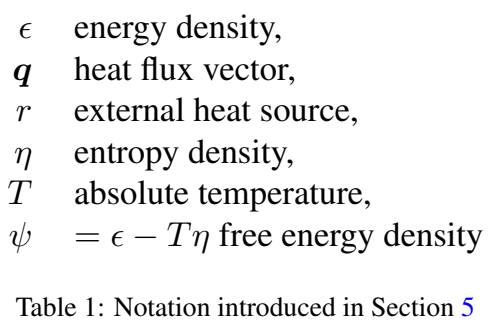

It is convenient to express the term $\boldsymbol{P} \cdot \dot{\boldsymbol{F}}$, which is the power expended due to deformation, in terms of the lattice Lagrangian strain $\mathbb{E}^{\mathrm{L}}$ as

$$
\begin{aligned}
\boldsymbol{P} \cdot \dot{\boldsymbol{F}} & =\boldsymbol{S} \cdot \dot{\mathbb{E}}^{\mathrm{L}}+\sum_{\alpha=1}^{A}\left(\psi, \mathbb{E}^{\mathrm{L}} \boldsymbol{m}^{\alpha} \cdot \boldsymbol{C}^{\mathrm{L}} \boldsymbol{s}^{\alpha}\right) v^{\alpha} \\
& =\boldsymbol{S} \cdot \dot{\mathbb{E}}^{\mathrm{L}}+\sum_{\alpha=1}^{A} \tau^{\alpha} v^{\alpha}
\end{aligned}
$$

where

$$
\boldsymbol{S}:=\boldsymbol{F}^{\mathrm{L}-1} \boldsymbol{P} \boldsymbol{F}^{\mathrm{pT}}, \quad \tau^{\alpha}:=\psi, \mathbb{E}^{\mathrm{L}} \boldsymbol{m}^{\alpha} \cdot \boldsymbol{C}^{\mathrm{L}} \boldsymbol{s}^{\alpha}
$$

are the lattice stress tensor and the resolved shear stress on the $\alpha$ slip plane respectively. The second law is written in the form of the Clausius-Duhem inequality for an arbitrary subpart $\mathcal{P}^{0}$ as

$$
\overline{\int_{\mathcal{P}^{0}} \eta d V} \geq-\int_{\partial \mathcal{P}^{0}} \frac{\boldsymbol{q} \cdot \boldsymbol{n}}{T} d A+\int_{\mathcal{P}^{0}} \frac{r}{T} d V
$$

where $\eta(\boldsymbol{X}, t)$ and $T(\boldsymbol{X}, t)$ denote the entropy density and temperature fields respectively. This implies

$$
\dot{\eta} \geq-\operatorname{Div}\left(\frac{\boldsymbol{q}}{T}\right)+\frac{r}{T}
$$

Multiplying the above equation by $T$ results in

$$
\begin{aligned}
T \dot{\eta} & \geq-\operatorname{Div} \boldsymbol{q}+\frac{\boldsymbol{q} \cdot \nabla T}{T}+r \\
& =\dot{\epsilon}-\left(\boldsymbol{S} \cdot \dot{\mathbb{E}}^{\mathrm{L}}+\mathfrak{p} \cdot \nabla \dot{\phi}\right)-\sum_{\alpha}\left(\Pi^{\alpha} v^{\alpha}+\tau^{\alpha} v^{\alpha}+\boldsymbol{\xi}^{\alpha} \cdot \nabla v^{\alpha}\right)-\pi \dot{\phi}+\frac{\boldsymbol{q} \cdot \nabla T}{T}
\end{aligned}
$$

where to arrive at the last equality, we replaced Div $\boldsymbol{q}$ using the relation given in (23). Introducing the free energy density $\psi=\epsilon-T \eta$ into the above equation, we obtain the following dissipation inequality:

$$
\dot{\psi}+\dot{T} \eta-\left(\boldsymbol{S} \cdot \mathbb{E}^{\mathrm{L}}+\mathfrak{p} \cdot \nabla \dot{\phi}\right)-\sum_{\alpha}\left(\Pi^{\alpha} v^{\alpha}+\tau^{\alpha} v^{\alpha}+\boldsymbol{\xi}^{\alpha} \cdot \nabla v^{\alpha}\right)-\pi \dot{\phi}+\frac{\boldsymbol{q} \cdot \nabla T}{T} \leq 0 .
$$

\section{Constitutive equations and the Coleman-Noll procedure}

In this section, we arrive at thermodynamically-consistent constitutive laws that connect the forces introduced in Section 4 to the kinematic variables introduced in Section 3.1 using the Coleman-Noll procedure. The guiding principle here is to include grain boundary energy in addition to the bulk elastic energy into the free energy density $\psi$ through its dependence on $s=\left(T, \nabla T, \mathbb{E}^{\mathrm{L}}, \boldsymbol{G}, \phi, \dot{\phi}, \nabla \phi\right), \boldsymbol{v}=\left(v^{1}, \ldots, v^{\alpha}\right)$ and $\nabla \boldsymbol{v}=\left(\nabla v^{1}, \ldots, \nabla v^{\alpha}\right)$. We begin with the following constitutive assumptions:

$$
\psi=\widehat{\psi}(s, \boldsymbol{v}, \nabla \boldsymbol{v})
$$


and the fields $\eta, \boldsymbol{q}, \boldsymbol{\xi}^{\alpha}, \pi, \Pi^{\alpha}$ and $\boldsymbol{S}$ are assumed to be functions of $s, \boldsymbol{v}, \nabla \boldsymbol{v}$ and $\boldsymbol{F}^{\mathrm{P}}$. The dependence of the free energy on $\mathbb{E}^{\mathrm{L}}$ and $\boldsymbol{G}$ instead of $\boldsymbol{F}^{\mathrm{L}}, \boldsymbol{F}^{\mathrm{P}}$ or $\nabla \boldsymbol{F}^{\mathrm{P}}$ is a result of the frame-invariance of $\psi$ [41].

Using the Coleman-Noll procedure, demonstrated in Appendix B, we arrive at the following restrictions on the above functional forms that make them thermodynamically-consistent. First, $\psi$ does not depend on $\nabla T, \dot{\phi}, \boldsymbol{v}$ or $\nabla \boldsymbol{v}$, and

$$
\begin{aligned}
& \eta=-\psi, T, \\
& \boldsymbol{S}=\psi, \mathbb{E}^{\mathrm{L}}, \\
& \mathfrak{p}=\psi, \nabla \phi .
\end{aligned}
$$

Second, the microscopic stress $\boldsymbol{\xi}^{\alpha}$ consists of an energy part $\boldsymbol{\xi}_{\mathrm{en}}^{\alpha}$, and a dissipative part $\boldsymbol{\xi}_{\mathrm{d}}^{\alpha}$, i.e.

$$
\boldsymbol{\xi}^{\alpha}=\boldsymbol{\xi}_{\mathrm{en}}^{\alpha}+\boldsymbol{\xi}_{\mathrm{d}}^{\alpha} .
$$

$\xi_{\text {en }}^{\alpha}$ can be interpreted as the distributed Peach-Koehler force due to the pile up of dislocations, and it is given by

$$
\boldsymbol{\xi}_{\text {en }}^{\alpha}=J^{\mathrm{P}}\left(\boldsymbol{F}^{\mathrm{P}}\right)^{-1}\left(\boldsymbol{m}^{\alpha} \wedge \psi, \boldsymbol{G} \boldsymbol{s}^{\alpha}\right),
$$

where $J^{\mathrm{P}}:=\operatorname{det} \boldsymbol{F}^{\mathrm{P}}$. The term $\boldsymbol{\xi}_{\mathrm{d}}^{\alpha}$ is the dissipative microstress conjugate to the gradient in slip rate, and given by

$$
\boldsymbol{\xi}_{\mathrm{d}}^{\alpha}=B^{\alpha} \nabla v^{\alpha},
$$

where $B^{\alpha}$ is a positive-valued inverse mobility associated to $\nabla \boldsymbol{v}$. Finally, the scalar internal microforces $\Pi^{\alpha}$ and $\pi^{\alpha}$, and the heat flux vector $\boldsymbol{q}$ are given by

$$
\begin{aligned}
\Pi^{\alpha} & =\psi,_{G}:\left(\mathbb{S}^{\alpha} \boldsymbol{G}+\boldsymbol{G} \mathbb{S}^{\alpha \mathrm{T}}\right)-\tau^{\alpha}+b^{\alpha}(s, \boldsymbol{v}, \nabla \boldsymbol{v}) v^{\alpha} \\
\boldsymbol{q} & =-\boldsymbol{K}(s, \boldsymbol{v}, \nabla \boldsymbol{v}) \nabla T \\
\pi & =\psi,_{\phi}+b^{\phi}(s, \boldsymbol{v}, \nabla \boldsymbol{v}) \dot{\phi}
\end{aligned}
$$

where the functions $b^{\alpha}$ and $b^{\phi}$ are positive-valued inverse mobilities associated to the slip rate $v^{\alpha}$ and $\dot{\phi}$ respectively, and $\boldsymbol{K}$ is the thermal conductivity tensor. Summarising, we have now expressed all forces appearing in the governing equations 19, 20, 21 and 23 in terms of the kinematic variables, temperature and its gradient.

\subsection{Bulk and grain boundary energies}

In this section, we construct an explicit free energy density $\psi\left(T, \mathbb{E}^{\mathrm{L}}, \boldsymbol{G}, \phi, \nabla \phi\right)$ such that it includes temperaturedependent bulk elastic and grain boundary energies. We assume that $\psi$ is additively decomposed into bulk elastic and grain boundary energy densities, given by

$$
\psi=\psi^{\mathrm{b}}\left(T, \mathbb{E}^{\mathrm{L}}\right)+\psi^{\mathrm{gb}}(T, \phi, \nabla \phi, \boldsymbol{G}),
$$

where we assume that $\psi^{\mathrm{b}}$ is the classical elastic energy that depends only on $T$ and $\mathbb{E}^{\mathrm{L}}$, while $\psi^{\text {gb }}$ is independent of $\mathbb{E}^{\mathrm{L}}$. For $\psi^{\mathrm{gb}}$, we use the following polyconvex energy density for isotropic materials proposed by Ciarlet and Geymonat [42],

$$
\begin{aligned}
\psi^{\mathrm{b}}= & a\left(3+2 \operatorname{Tr}\left(\mathbb{E}^{\mathrm{L}}\right)\right)+b\left(3+4 \operatorname{Tr}\left(\mathbb{E}^{\mathrm{L}}\right)+2 \operatorname{Tr}^{2}\left(\mathbb{E}^{\mathrm{L}}\right)-2 \operatorname{Tr}\left(\left(\mathbb{E}^{\mathrm{L}}\right)^{2}\right)\right)+ \\
& c \operatorname{det}\left(2 \mathbb{E}^{\mathrm{L}}+\boldsymbol{I}\right)-\frac{1}{2} d \log \operatorname{det}\left(2 \mathbb{E}^{\mathrm{L}}+\boldsymbol{I}\right)-(3 a+3 b+c),
\end{aligned}
$$

where the constants $a, b, c$ and $d$ are expressed in terms of the isotropic materials' Lamé constants $\lambda$ and $\mu$ as.

$$
\begin{aligned}
& a=\mu+\frac{1}{2}\left(-\mu-\frac{\lambda}{4}\right), \quad b=-\frac{\mu}{2}-\frac{1}{2}\left(-\mu-\frac{\lambda}{4}\right), \\
& c=\frac{\lambda}{8}, \quad d=\mu+\frac{\lambda}{2} .
\end{aligned}
$$

The construction of $\psi^{\mathrm{gb}}$ is the most non-trivial part of the constitutive law. Under the kinematic framework developed in Section 3.1, since the initial distribution of GNDs describe grain boundaries, it is natural to develop a grain boundary 
energy density that is a function of $\boldsymbol{G}$. Moreover, we expect that a steady state solution yields a grain boundary of finite width, a feature central to the KWC model. Therefore, the construction of $\psi^{\mathrm{gb}}$ is inspired by the KWC energy functional given in (2). Recall that the order parameter $\theta$ in the KWC energy functional describes the orientation of the lattice. Therefore, we intend to construct $\psi^{\text {gb }}$ by replacing $\nabla \theta$ in (2) with the gradient of the lattice orientation. It is well-known that the GND tensor $\boldsymbol{G}$ describes the gradient of lattice rotations under the assumption of small strain gradients [43]. To the best of our knowledge, the only relation that connects the gradient of lattice rotation to the GND tensor and the lattice strain exists under geometric linearity, attributed to Kröner et al. [44]. In Appendix C, we show under a geometrically nonlinear setting, that the gradient of lattice rotation can be expressed in terms of the GND tensor, the lattice stretch tensor, and its gradient as

$$
\boldsymbol{R}^{\mathrm{LT}}\left(\operatorname{curl} \boldsymbol{R}^{\mathrm{LT}}\right)=\left(J^{\mathrm{L}}\right)^{-1}\left(\boldsymbol{U}^{\mathrm{L}} \boldsymbol{G} \boldsymbol{U}^{\mathrm{LT}}+\boldsymbol{U}^{\mathrm{L}} \overline{\operatorname{curl}} \boldsymbol{U}^{\mathrm{L}}\right)
$$

where $\overline{c u r l}$ and curl denote the curl operators with respect to the lattice and deformed configurations respectively.

The term $\boldsymbol{R}^{\mathrm{LT}}$ (curl $\boldsymbol{R}^{\mathrm{LT}}$ ) in (39), which describes the gradient of lattice rotation, qualifies to replace $\nabla \theta$ in the KWC energy density due to its frame-indifference. ${ }^{9}$ The resulting $\psi^{\text {gb }}$ is a function of $\boldsymbol{G}, \boldsymbol{U}^{\mathrm{L}}$ and its gradient, yielding a lattice strain gradient model. ${ }^{10}$ In this paper, we do not pursue such a model in the interest of computational simplicity. ${ }^{11}$ Instead, we construct $\psi^{\text {gb }}$ by replacing $\nabla \theta$ in (2) with $G$, i.e.

$$
\psi^{\mathrm{gb}}(T, \phi, \nabla \phi, \boldsymbol{G})=\frac{\alpha^{2}}{2}|\nabla \phi|^{2}+f(\phi)+g(\phi) s|\boldsymbol{G}|+\frac{\epsilon^{2}}{2}|\boldsymbol{G}|^{2},
$$

where $\alpha$ and $\epsilon$ are functions of $T$. In Section 7.3, we show that although (40) is not an exact analog of the KWC energy density, it results in the intended grain boundary motion by curvature. Moreover, the dependence of $\psi^{\mathrm{gb}}$ on the norm of $G$ results in a free energy density that depends only on the misorientation, and not on the inclination of a grain boundary. We defer to future work any generalization to include the dependence of free energy on inclination.

Recall that the kinematics described in Section 3.1 enables us to construct lattice strain-free sharp- or diffuseinterface grain boundaries. It is clear from (40) that $\psi^{\mathrm{gb}}$ for a sharp-interface grain boundary is infinite. Therefore, similar to the KWC model, we expect that the steady state solution to the governing equations result in a finite grain boundary thickness which depends on the parameters $\epsilon$ and $\alpha$.

\section{Results}

The goal of this section is to demonstrate that the polycrystal plasticity model presented in this paper can simulate the four elementary grain boundary processes: (i) grain boundary sliding and coupled motions, (ii) grain rotation, (iii) grain shrinkage, and (iv) interactions between bulk dislocations and grain boundaries. Although the framework developed in this paper is applicable to an arbitrary polycrystal in any dimension, we limit our numerical study to bicrystals in one and two dimensions since our primary focus at this stage is to demonstrate the validity of the model rather than apply to study problems in crystal plasticity. In addition, we do not solve the energy balance equation in this numerical study as all simulations are performed at a constant temperature.

The Lamé constants entering the bulk elastic energy density (see (37) and (38)) are taken as $\lambda=9.515 \times 10^{-2} \mathrm{fJ} \mathrm{nm}^{-3}$ and $\mu=4.477 \times 10^{-2} \mathrm{fJ} \mathrm{nm}{ }^{-3}$. The presence of the linear term $g(\phi) s|\boldsymbol{G}|$ in (40), results in a singular diffusive term in the microscopic balance equation (20). Therefore, similar to the KWC model, a numerical implementation of the model warrants approximating $|\boldsymbol{G}|$ in $g(\phi) s|\boldsymbol{G}|$ with $p(\boldsymbol{G})$, where the function $p$ is defined in (4). The material parameters corresponding to the grain boundary energy density are identical to those used to simulate the KWC model (see Table A.5), unless otherwise stated. The parameter $B^{\alpha}$ associated with the dissipative microstress $\boldsymbol{\xi}_{\mathrm{d}}^{\alpha}$ (see (34)) is equal to $1 \mathrm{fJ} \mathrm{s} \mathrm{m}^{-1}$ for all the simulations. We assume that plastic distortion evolves due to the presence of four slip

\footnotetext{
${ }^{9}$ Note that the term $\boldsymbol{R}^{\mathrm{LT}}$ ( $\operatorname{curl} \boldsymbol{R}^{\mathrm{LT}}$ ) maps the lattice configuration to itself, and since the vectors and tensors defined in the lattice configuration are frame-indifferent, the resulting $\psi^{\mathrm{gb}}$ is frame-invariant.

${ }^{10}$ Note the distinction between a lattice strain gradient theory and the more commonly used theory of "strain gradient plasticity", where gradient in the latter refers to the gradient in $\boldsymbol{F}^{\mathrm{P}}$.

${ }^{11}$ A lattice strain gradient theory of grain boundaries would involve modifying the principle of virtual work, stated in Section 4 , to include power expended due to the kinematic variable $\nabla \boldsymbol{F}$ and its corresponding hyperstress.
} 


\begin{tabular}{|c|c|}
\hline Div $\boldsymbol{P}=\mathbf{0}$ in $\mathcal{B}^{0}$ & \\
with Dirichlet boundary condition on $\boldsymbol{u}$ & $\begin{array}{c}\text { Div } \boldsymbol{\xi}^{\alpha}-\Pi^{\alpha}=0 \text { in } \mathcal{B}^{0} \\
v^{\alpha}\left(\partial \mathcal{B}^{0}, t\right)\end{array}$ \\
& \\
\hline & \\
$\operatorname{Div} \mathfrak{p}-\pi=0$ in $\mathcal{B}^{0}$ & $\dot{\boldsymbol{F}}^{\mathrm{P}}=\boldsymbol{L}^{\mathrm{P}} \boldsymbol{F}^{\mathrm{P}}$ in $\mathcal{B}^{0}$ \\
$\phi\left(\mathcal{B}^{0}, 0\right) \equiv 1$ & $\boldsymbol{F}^{\mathrm{P}}(\cdot, 0)=\boldsymbol{R}^{0 \mathrm{~T}}$ in $\mathcal{B}^{0}$ \\
$\phi\left(\partial \mathcal{B}^{0}, t\right) \equiv 1$ & \\
\hline
\end{tabular}

Table 2: The governing equations for the Dirichlet BVP for the unknown kinematic variables $\boldsymbol{u}, v^{\alpha}(\alpha=1, \ldots, A), \phi$ and $\boldsymbol{F}^{\mathrm{P}}$. The forces $\boldsymbol{P}$, $\mathfrak{p}, \boldsymbol{\xi}^{\alpha}, \Pi$ and $\pi$ are expressed in terms of the kinematic variables through the relations given in (31)-(35), with $\boldsymbol{F}^{\mathrm{L}}=\boldsymbol{F} \boldsymbol{F}^{\mathrm{p}-1}$. The short-hand argument $\left(\partial \mathcal{B}^{0}, t\right)$ is used to described a function on $\partial \mathcal{B}^{0}$ at time $t$.

systems, i.e. $A=4$ in all the simulations. The slip directions are taken as

$$
\begin{aligned}
& s^{1}=(1,0), \\
& s^{2}=(0,1), \\
& s^{3}=\frac{1}{\sqrt{2}}(1,1), \\
& s^{4}=\frac{1}{\sqrt{2}}(-1,1),
\end{aligned}
$$

with the corresponding normals perpendicular to the slip direction and the out-of-plane dislocation line direction. In simulations where we want only a subset of the above-mentioned slip systems, we deactivate the remaining slip systems by decreasing the corresponding mobilities.

The initial bicrystal is constructed using a step function $\theta(\boldsymbol{X})$ representing the orientation of the crystal lattice. As described in Section 3.1, a diffuse-interface polycrystal is generated by regularizing $\theta(\boldsymbol{X})$ into a smooth function $\widetilde{\theta}(\boldsymbol{X})$, and starting with the initial condition $\boldsymbol{F}^{\mathrm{P}}(\boldsymbol{X}, 0)=\widetilde{\boldsymbol{R}}^{0 \mathrm{~T}}(\boldsymbol{X})$, where $\widetilde{\boldsymbol{R}}^{0}$ is the smooth field in $S O(3)$ corresponding to $\widetilde{\theta}(\boldsymbol{X})$. The governing equations with Dirichlet boundary conditions, listed in Table 2, are numerically solved for the unknowns $\boldsymbol{u}, v^{\alpha}(\alpha=1, \ldots, A), \phi$ and $\boldsymbol{F}^{\mathrm{P}}$ using the finite element method.

The three displacement variables $u_{1}, u_{2}$ and $u_{3}$, the four slips $v_{i}(i=1, \ldots, 4)$, and the order parameter $\phi$ are interpolated using the Lagrange quadratic finite elements. Since $\boldsymbol{F}^{\mathrm{P}}$ is a smooth rotation field at $t=0$, it satisfies the orthogonality condition $\boldsymbol{F}^{\mathrm{pT}} \boldsymbol{F}^{\mathrm{P}} \equiv \boldsymbol{I}$. However, because a Lagrange finite element interpolation of $\boldsymbol{F}^{\mathrm{P}}$ does not satisfy such condition, we express $\boldsymbol{F}^{\mathrm{P}}$ using its polar decomposition $\boldsymbol{F}^{\mathrm{P}}=\boldsymbol{R}^{\mathrm{P}} \boldsymbol{U}^{\mathrm{P}}$, where $\boldsymbol{R}^{\mathrm{P}} \in S O(3)$, and $\boldsymbol{U}^{\mathrm{P}}$ is the positive-definite symmetric stretch tensor. Moreover, since we are limiting ourselves to at most two dimensions, $\boldsymbol{R}^{\mathrm{P}}$ is a function of a single variable $\theta^{\mathrm{P}}$. Using the above representation, $\boldsymbol{F}^{\mathrm{P}}$ is interpolated using the Lagrange quadratic finite element interpolation of $\theta^{\mathrm{P}}, U_{11}^{\mathrm{P}}, U_{12}^{\mathrm{P}}$ and $U_{22}^{\mathrm{P}}$. This guarantees the interpolant of $\boldsymbol{F}^{\mathrm{P}}(\boldsymbol{X}, 0)$ to be in $S O(3)$.

The system of equations listed in Table 2 is evolved in a segregated manner using the MUMPS direct solver, and BDF (Backward Differential Formula) time stepping algorithm implemented in COMSOL 5.2.

\subsection{Steady state solution of a flat grain boundary}

In this section, we present a simulation of a symmetric tilt grain boundary in a bicrystal modeled as a onedimensional domain $\Omega=[0, L]$. The aim of this simulation is to study the steady state solution corresponding to a flat grain boundary, and the corresponding energy as a function of the misorientation angle.

The unknowns here are the two displacements $u_{1}$ and $u_{2}$, the four slip rates, the order parameter $\phi$, and the four components $F_{11}^{\mathrm{P}}, F_{12}^{\mathrm{P}}, F_{21}^{\mathrm{P}}$, and $F_{22}^{\mathrm{P}}$ of $\boldsymbol{F}^{\mathrm{P}}$. The system is initialized as:

$$
\boldsymbol{u}\left(X_{1}, 0\right)=\mathbf{0}, \quad \phi\left(X_{1}, 0\right)=1, \quad \boldsymbol{F}^{\mathrm{P}}\left(X_{1}, 0\right)=\boldsymbol{R}^{0 \mathrm{~T}}\left(\widetilde{\theta}\left(X_{1}\right)\right),
$$




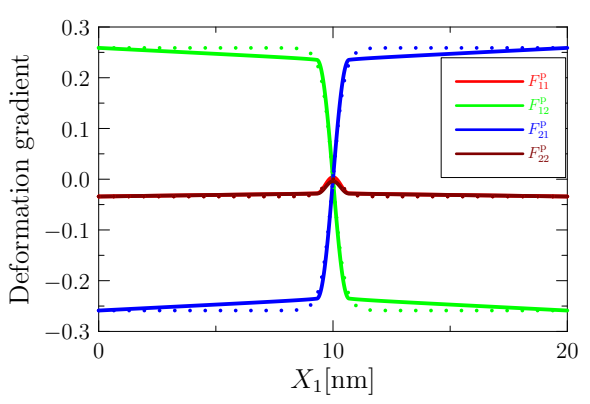

(a) Plastic distortion

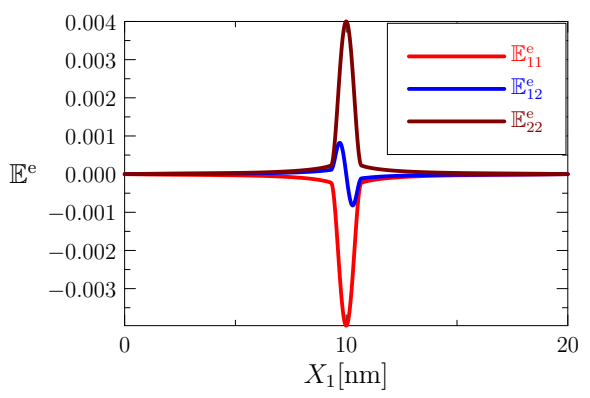

(c) Lattice Lagrangian strain

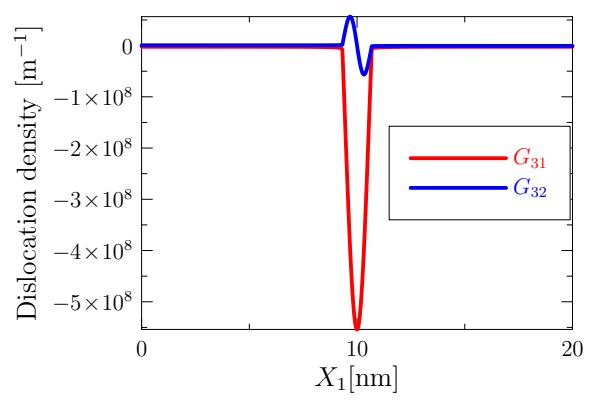

(b) Dislocation density

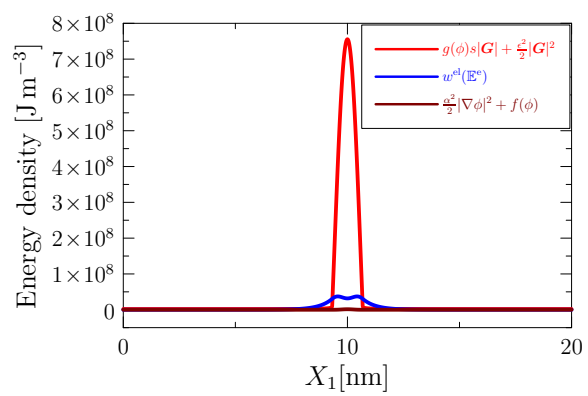

(d) Constributions to the total energy density

Figure 4: Plots of the 1-d simulation modeling a flat grain boundary under steady state with $g(\phi)=\phi^{2}$, and material parameters as listed in Table A.5.

\begin{tabular}{|c|l|}
\hline$\epsilon^{2}$ & $3.1999 \times 10^{-4} \mathrm{fJ} \mathrm{nm}^{-1}$ \\
$\alpha^{2}$ & $7.95 \times 10^{-3} \mathrm{fJ} \mathrm{nm}^{-1}$ \\
$s$ & $0.0085 \mathrm{fJ} \mathrm{nm}^{-2}$ \\
$e$ & $0.00035 \mathrm{fJ} \mathrm{nm}^{-3}$ \\
$L$ & $20 \mathrm{~nm}$ \\
\hline
\end{tabular}

Table 3: Parameters used in the implementation of the current model with $g(\phi)=-2(\log (\phi-1)-\phi)$.

where

$$
\widetilde{\theta}\left(X_{1}\right)=-\frac{\theta_{0}}{2}+\frac{\theta_{0}}{\left(1+\exp \left(-2.5\left(X_{1}-10\right)\right)\right)},
$$

and $\boldsymbol{R}^{0}(\widetilde{\theta})$ is the rotation corresponding to $\widetilde{\theta}$. Equation (43) describes a diffuse-interface grain boundary at $t=0$ with the lattice orientation changing smoothly from $-\theta_{0} / 2$ to $\theta_{0} / 2$ across the grain boundary. The boundary conditions are given by

$$
\phi(0, t)=\phi(L, t)=1, \quad \boldsymbol{v}(0, t)=\boldsymbol{v}(L, t)=\mathbf{0},
$$

which indicate perfect crystalline order, and no slip at the boundaries. The inverse mobilities $b^{\alpha}(\alpha=1, \ldots, A)$ and $b^{\phi}$, corresponding to the slip rates and $\phi$ respectively, are assumed to be constant, and equal to 1 (fJ ns)/nm ${ }^{3}$. The system is evolved for $10^{-5} \mathrm{~ns}$, long enough to reach a steady state.

Fig. 4 shows the plots obtained at the end of the simulation. We make the following observations on the steady state solution:

1. Fig. 4a shows the plots of the four components of $\boldsymbol{F}^{\mathrm{P}}$, with dotted lines corresponding to the initial condition. From the plots we observe that the gradient $\boldsymbol{F}^{\mathrm{P}}$ is not identically equal to zero in the interior of the grains. This is a result of approximating $|\boldsymbol{G}|$ in the term $g(\phi) s|\boldsymbol{G}|$ of (40) with $p(|\boldsymbol{G}|)$, an approximation used in the numerical implementation of the KWC model, as noted in Section 2. 


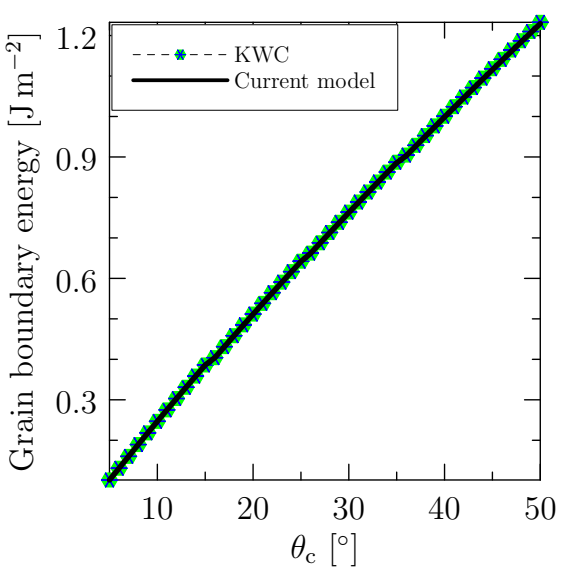

(a) $g(\phi)=\phi^{2}$

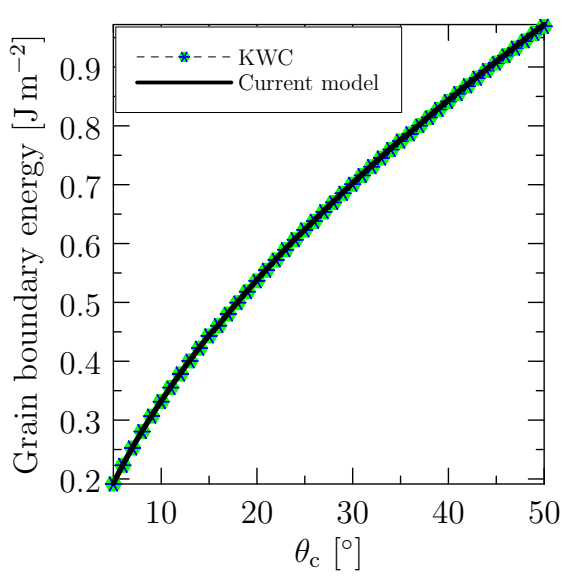

(b) $g(\phi)=-2(\log (\phi-1)-\phi)$

Figure 5: Plot of the grain boundary energy versus misorientation for different choices of $g$.

2. Fig. 4 b shows the plots of the non-zero components $G_{31}$ and $G_{32}$ of $\boldsymbol{G}$. As noted in eq. (15), a non-zero $G_{32}$ in a symmetric tilt grain boundary signifies the diffuse nature of the grain boundary.

3. The plots of the three components of $\mathbb{E}^{\mathrm{L}}$ in Fig. $4 \mathrm{c}$, show the presence of a maximum strain of $0.4 \%$ at steady state. $^{12}$ On the other hand, in real materials with no defects (other than grain boundaries) under no external loads, the lattice Lagrangian strain is oscillatory about zero in the vicinity of the grain boundary. In the current model, with grain boundaries represented by a continuum dislocation distribution -as opposed to a discrete array of dislocations-, no Lagrangian strain is expected near the grain boundaries. The presence of a nonzero $\mathbb{E}^{\mathrm{L}}$ can be attributed to the construction $\psi_{\mathrm{gb}}$ (see (40)), which uses $\boldsymbol{G}$ as opposed to the exact lattice gradient tensor $\boldsymbol{R}^{\mathrm{LT}} \operatorname{curl} \boldsymbol{R}^{\mathrm{LT}}$ discussed at the end of Section 6.

4. Fig. 4d shows the plots of the three parts of the total energy density. It is clear that the major contribution is due to the GND density, followed by the elastic contribution and then the contribution due to the order parameter $\phi$.

5. In the KWC model, the approximation in eq. (4) is used for numerical convenience, to tackle the singular diffusive term. However, the approximate functional -in particular the length constant $\gamma$ - has an interesting physical interpretation in the current model. As $\gamma$ increases the gradient of the plastic distortion tends to zero in the bulk, resulting in negligible dislocation density in the bulk. Therefore, $\gamma$ can be interpreted as the propensity of bulk dislocations to agglomerate and form grain boundaries. This effect will be discussed in more depth in Section 7.4

Next, we study the variation of grain boundary energy with respect to misorientation. Kobayashi and Giga [36] have noted that $g(\phi)=\phi^{2}$ results in a linear dependence of the grain boundary energy on misorientation, while $g(\phi)=$ $-2(\log (\phi-1)-\phi)$ results in non-convex grain boundary energy as predicted by Read and Shockley [45]. Our model confirms this observation as well, as shown in Fig. 5, from simulations performed with the material parameters given in Table 3. In fact, the figure shows an excellent agreement between the energy predicted by the KWC model and the current polycrystal plasticity model for the different choices of $g$. In addition, it also reveals the following limitation: although the current model is kinematically nonlinear, i.e. a grain boundary with an arbitrary misorientation can be constructed, the energy functional cannot detect the equivalence of zero and $90^{\circ}$ misorientations. Therefore, the energy monotonically increases as the magnitude of the misorientation increases. This limitation appears to arise due to the diffuse-interface nature of grain boundaries.

\subsection{Shear-induced grain boundary motion}

The goal of this section is to model the motion of a flat grain boundary in a bicrystal subjected to shear stress. The absence of curvature in this simulation results in stress being the only driving force for plastic distortion.

Various experimental and molecular dynamics simulations have identified two different mechanisms by which a bicrystal plastically deforms when subjected to a shear stress. In the first mechanism, commonly referred to as

${ }^{12}$ Recall that at $t=0$, the lattice Lagrangian strain is zero since $\boldsymbol{F}^{\mathrm{L}}(\boldsymbol{X}, 0) \in S O(3)$. 


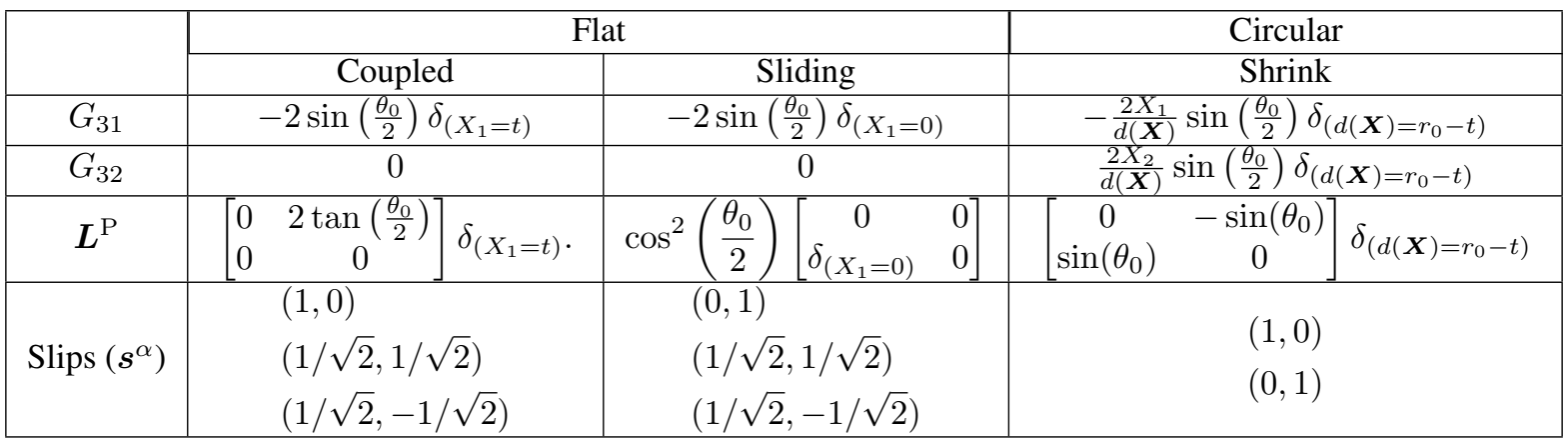

Table 4: A catalog of time-dependent GND tensor and $\boldsymbol{L}^{\mathrm{P}}$ fields for the motion of a flat and circular sharp-interface grain boundaries. The three columns correspond to the evolution of coupled and sliding flat grain boundaries, and a shrinking circular grain boundary. The last row lists the slip systems used to simulate the three cases.

"coupled", the flat grain boundary translates perpendicular to its normal, plastically distorting (i.e. $\boldsymbol{F}^{\mathrm{P}} \not \equiv \boldsymbol{I}$ ) and macroscopically deforming (i.e. $\boldsymbol{F} \not \equiv \boldsymbol{I}$ ) the material in the swept volume. In addition, $\boldsymbol{F}$ and $\boldsymbol{F}^{\mathrm{P}}$ are uniquely determined by the misorientation of the bicrystal. On the other hand, in the second mechanism commonly called "sliding", the grain boundary remains stationary while the two grains slide with respect to each other tangential to the grain boundary, resulting in a plastic distortion concentrated on the grain boundary. We now give a precise definition of a coupled motion of a symmetric-tilt grain boundary, followed by grain boundary sliding. We use the notation $\mathbb{1}_{A}(x, t)$ where $x \in \mathbb{R}^{d}(d=1,2)$ and $A \subset \mathbb{R}^{d+1}$, to denote the indicator function defined as

$$
\mathbb{1}_{A}(x, t)=\left\{\begin{array}{l}
1 \text { if }(x, t) \in A, \\
0 \text { otherwise },
\end{array}\right.
$$

and $\delta_{x}$ to denote a Dirac delta distribution with support at $x$. We also use the notation $(\square)$ to denote a set whose elements satisfy the inequality/equality $\square$.

Consider a bicrystal defined on a one-dimensional domain $\mathcal{B}=[-L, L]$, with:

$$
\boldsymbol{F}^{\mathrm{L}}=\mathbb{1}_{(X<0)} \boldsymbol{R}\left(\frac{\theta_{0}}{2}\right)+\mathbb{1}_{(X \geq 0)} \boldsymbol{R}\left(-\frac{\theta_{0}}{2}\right)
$$

and

$$
\boldsymbol{F}^{\mathrm{P}}=\left(\boldsymbol{F}^{\mathrm{L}}\right)^{-1} \text {. }
$$

In other words, $\mathcal{B}$ is a lattice strain-free bicrystal, with the symmetric-tilt grain boundary at $X=0$.

Definition 1 (Coupled grain boundary motion). A time-dependent coupled grain boundary motion in the bicrystal $\mathcal{B}$ is given by the following plastic and elastic distortion fields:

$$
\begin{aligned}
& \boldsymbol{F}^{\mathrm{L}}\left(X_{1}, t\right)=\mathbb{1}_{\left(X_{1}<t\right)} \boldsymbol{R}\left(\frac{\theta_{0}}{2}\right)+\mathbb{1}_{\left(X_{1} \geq t\right)} \boldsymbol{R}\left(-\frac{\theta_{0}}{2}\right), \\
& \boldsymbol{F}^{\mathrm{P}}\left(X_{1}, t\right)=\mathbb{1}_{\left(X_{1}<0\right)} \boldsymbol{R}\left(-\frac{\theta_{0}}{2}\right)+\mathbb{1}_{\left(X_{1} \geq t\right)} \boldsymbol{R}\left(\frac{\theta_{0}}{2}\right)+\mathbb{1}_{\left(0 \leq X_{1}<t\right)} \boldsymbol{S} \boldsymbol{R}\left(\frac{\theta_{0}}{2}\right),
\end{aligned}
$$

where

$$
\boldsymbol{S}=\left[\begin{array}{cc}
1 & 2 \tan \left(\frac{\theta_{0}}{2}\right) \\
0 & 1
\end{array}\right]
$$

Moreover, the resulting deformation gradient $\boldsymbol{F}=\boldsymbol{F}^{\mathrm{L}} \boldsymbol{F}^{\mathrm{P}}$ is the gradient of the continuous deformation field given by

$$
u_{1} \equiv 0, \quad u_{2}\left(X_{1}, t\right)=2 \tan \left(\frac{\theta_{0}}{2}\right)\left(\mathbb{1}_{\left(0 \leq X_{1}<t\right)} X_{1}+\mathbb{1}_{\left(X_{1} \geq t\right)} t\right) .
$$


By construction, Definition 1 applies to a sharp-interface grain boundary. Equations (46), (47) and (49) can be appropriately mollified to yield an analogous definition for a diffuse-interface grain boundary. The time-dependent non-zero components of the GND tensor for a coupled grain boundary motion corresponding to the plastic distortion field in (47) is given in Table 4 . The expression for $G_{31}$ clearly shows that a coupled sharp-interface grain boundary motion involves the translation of a single family of necessary edge dislocations. Note that there was no mention of slip systems of the crystal until this point. The translation of GNDs is made possible by the availability of the crystal slip systems. This is seen by evaluating ${ }^{13} \boldsymbol{L}^{\mathrm{P}}=\boldsymbol{F}^{\mathrm{P}} \boldsymbol{F}^{\mathrm{p}-1}$ (see (8a)), and shown in Table 4. The expressions for $\boldsymbol{G}$ and $\boldsymbol{L}^{\mathrm{P}}$ shown in Table 4 suggest that the single slip system

$$
s=(1,0)
$$

is sufficient to translate the GNDs in a sharp-interface grain boundary. Conversely, we know from (15) that for a diffuseinterface grain boundary $G_{32} \not \equiv 0$. Therefore, in order for the grain boundary to translate, the GNDs corresponding to $G_{32}$ should undertake a mechanism resulting in an "apparent" dislocation climb, made possible by the availability of two additional slip systems shown in Table 4. The translation of GND corresponding to $G_{32}$ along with the grain boundary can be interpreted as dissociating into dislocations in the second and third slip systems, translating, and recombining. Such a mechanism has been proposed by Cahn et al. [46].

Definition 2 (Grain boundary sliding). A time-dependent grain boundary sliding in $\mathcal{B}$ is given by the following plastic and elastic distortion fields:

$$
\begin{aligned}
& \boldsymbol{F}^{\mathrm{L}}\left(X_{1}, t\right)=\mathbb{1}_{\left(X_{1}<0\right)} \boldsymbol{R}\left(\frac{\theta_{0}}{2}\right)+\mathbb{1}_{\left(X_{1} \geq 0\right)} \boldsymbol{R}\left(-\frac{\theta_{0}}{2}\right), \\
& \boldsymbol{F}^{\mathrm{P}}\left(X_{1}, t\right)=\left(\mathbb{1}_{\left(X_{1}<0\right)} \boldsymbol{R}\left(-\frac{\theta_{0}}{2}\right)+\mathbb{1}_{\left(X_{1} \geq 0\right)} \boldsymbol{R}\left(\frac{\theta_{0}}{2}\right)\right) \boldsymbol{S}
\end{aligned}
$$

where

$$
\boldsymbol{S}=\left[\begin{array}{cc}
1 & 0 \\
t \delta_{\left(X_{1}=0\right)} & 1
\end{array}\right]
$$

and $\delta_{\left(X_{1}=0\right)}$ is the Dirac delta distribution with support at the origin. The resulting deformation gradient given by

$$
\boldsymbol{F}=\boldsymbol{F}^{\mathrm{L}} \boldsymbol{F}^{\mathrm{P}}=\left[\begin{array}{cc}
1 & 0 \\
t \delta_{\left(X_{1}=0\right)} & 1
\end{array}\right]
$$

is the gradient of the following discontinuous deformation map

$$
u_{1} \equiv 0, \quad u_{2}\left(X_{1}, t\right)=t \mathbb{1}_{[0, L]} .
$$

Similar to Definition 1, the above described grain boundary sliding applies to a sharp-interface grain boundary. Equations 51, 52 and 55 can be appropriately mollified to yield an analogous definition for a diffuse-interface grain boundary. It is interesting to note that the non-zero component $G_{31}$ of the GND tensor for grain boundary sliding, shown in Table 4, is independent of time which means the grain boundary remains stationary. The expression for $\boldsymbol{L}^{\mathrm{P}}$, given in Table 4, clearly shows that the $2-1$ component is the only non-zero component. Note that this is applicable only for a sharp-interface symmetric tilt grain boundary. For a diffuse grain boundary, all components of $\boldsymbol{L}^{\mathrm{P}}$ are nonzero, although the $2-1$ component is the dominant one. Therefore, the slip system

$$
\boldsymbol{s}=(0,1)
$$

is responsible for sharp-interface symmetric tilt grain boundary sliding, and two additional slip systems, shown in Table 4, are necessary in the diffuse case. In general, in the presence of all four slips systems (see equations in 41), the motion of the grain boundary involves a combination of coupling and sliding motions.

\footnotetext{
${ }^{13}$ In order to compute $\dot{\boldsymbol{F}}^{\mathrm{P}}$, we note that $\dot{\mathbb{1}}_{\left(X_{1} \geq t\right)}=-\delta_{\left(X_{1}=t\right)}$. Moreover, to compute $\dot{\boldsymbol{F}}^{\mathrm{P}} \boldsymbol{F}^{\mathrm{p}-1}$, we define the product of a Dirac delta distribution $\delta_{(X=0)}$ and a function $f(X)$ with discontinuity at $X=0$ as $\frac{1}{2} \delta_{(X=0)}\left(f\left(0^{+}\right)+f\left(0^{-}\right)\right)$.
} 


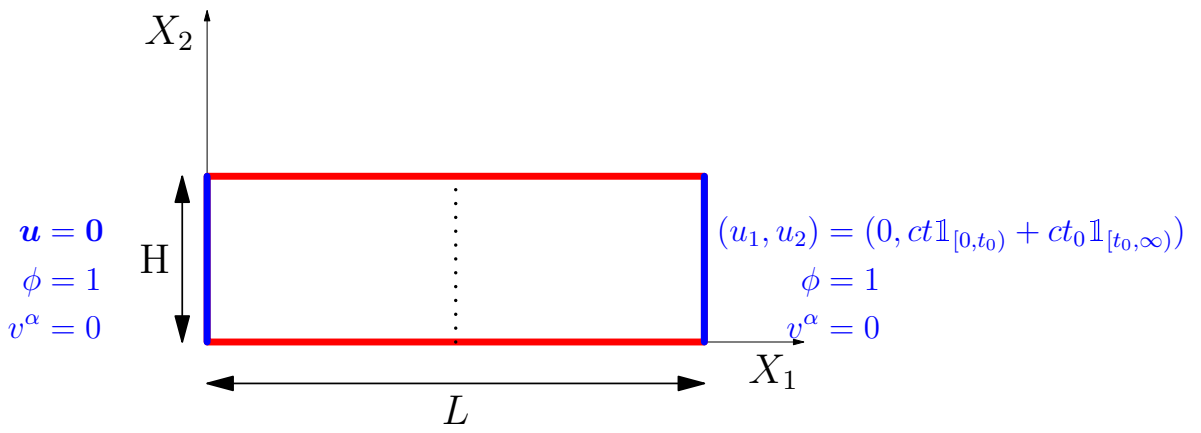

Figure 6: A schematic of a bicrystal used to study grain boundary sliding and coupled motion. The grain boundary is shown as a dotted line. Dirichlet boundary conditions are shown in blue, while periodic boundary conditions are shown in red. The right surface $X_{1}=L$ is translated upwards with a velocity $c$ for a time $t_{0}$, and subsequently held fixed in that position.

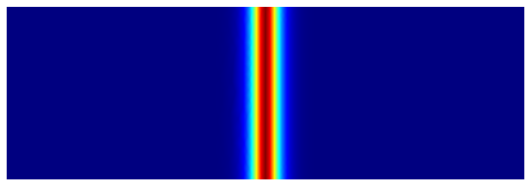

(a) $t=0 \mathrm{~s}$

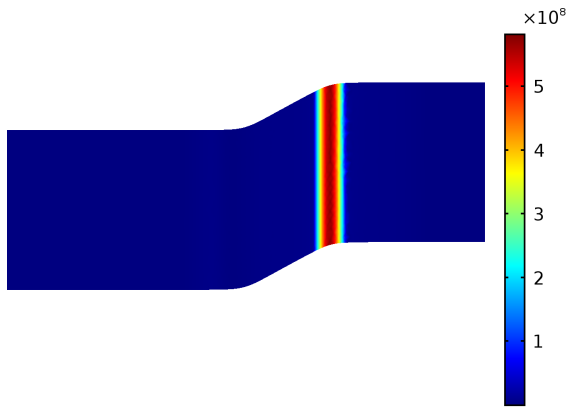

(b) $t=1 \times 10^{-4} \mathrm{~s}$

Figure 7: Coupled grain boundary motion: Color plots of the norm of the GND density in undeformed and the deformed configurations in units of $\mathrm{m}^{-1}$.

We now simulate grain boundary coupling and sliding in a rectangular bicrystal of size $L=20 \mathrm{~nm}$ and $H=$ $20 / 3 \mathrm{~nm}$, with a $30^{\circ}$ symmetric tilt grain boundary. The bicrystal, and the boundary conditions used in the simulation are depicted in Fig. 6. A shear stress is imposed on the bicrystal by translating the right surface $X_{1}=L$ upwards at a constant velocity $c=1 \times 10^{-4} \mathrm{~m} \mathrm{~s}^{-1}$ for a time $t_{0}=2 \times 10^{-5} \mathrm{~s}$, and then holding it in this position for the rest of the simulation. The initial conditions are taken to be

$$
\boldsymbol{u}(\boldsymbol{X}, 0)=\mathbf{0}, \quad \phi(\boldsymbol{X}, 0)=1, \quad \boldsymbol{F}^{\mathrm{P}}(\boldsymbol{X}, 0)=\boldsymbol{R}^{0 \mathrm{~T}}\left(\widetilde{\theta}\left(X_{1}\right)\right),
$$

where

$$
\widetilde{\theta}\left(X_{1}\right)=-\frac{\theta_{0}}{2}+\frac{\theta_{0}}{\left(1+\exp \left(-4\left(X_{1}-10\right)\right)\right.},
$$

$\theta_{0}=30^{\circ}$, and $\boldsymbol{R}^{0}(\widetilde{\theta})$ is the rotation corresponding to $\widetilde{\theta}$. We begin with the simulation of grain boundary coupled motion by having the three slip systems shown in Table 4 . The mobility for all slip systems is chosen as

$$
\left(b^{\alpha}\right)^{-1}=m_{\min }^{\alpha}+\left(1-\phi^{3}\left(10-15 \phi+6 \phi^{2}\right)\right)\left(m_{\max }^{\alpha}-m_{\min }^{\alpha}\right),
$$

where $m_{\min }^{\alpha}=1 \times 10^{-9} \mathrm{~nm}^{3} \mathrm{fJ}^{-1} \mathrm{~ns}^{-1}$ and $m_{\max }^{\alpha}=1 \mathrm{~nm}^{3} \mathrm{fJ}^{-1} \mathrm{~ns}^{-1}$ are the minimum and maximum mobilities attained when $\phi=1$ and $\phi=0$ respectively. In other words, (59) is constructed such that the material shows greater resistance to slip in the bulk compared to the grain boundary which is in agreement with experimental observations. ${ }^{14}$ The mobility corresponding to the order parameter $\phi$ is chosen as $\left(b^{\phi}\right)^{-1}=1 \mathrm{~nm}^{3} /$ (fJ ns).

\footnotetext{
${ }^{14}$ The construction of mobilities in (59) also ensures that the dislocations are more likely to nucleate in the grain boundary compared to the bulk.
} 

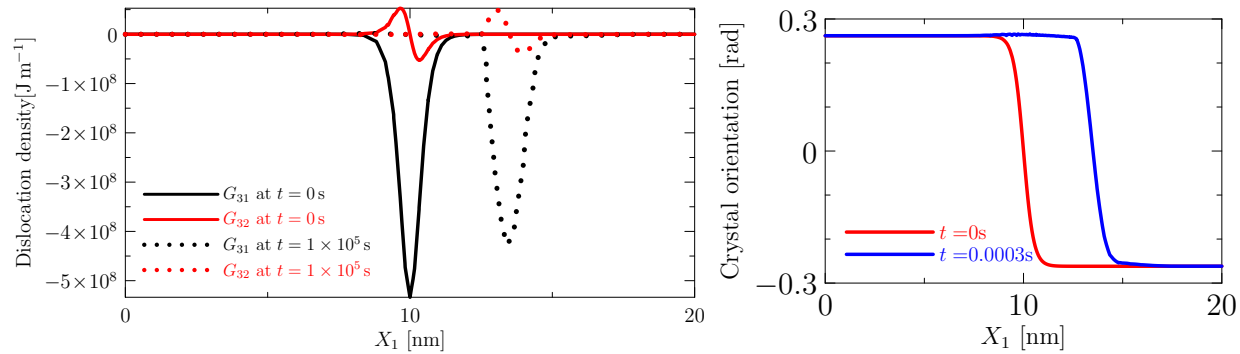

Figure 8: Coupled grain boundary motion: (a) Plots of GND density components $G_{31}$ and $G_{32}$, and (b) plots of the lattice orientation corresponding to the rotation $\boldsymbol{R}^{\mathrm{L}}$ obtained from the polar decomposition of $\boldsymbol{F}^{\mathrm{L}}$, along the horizontal central line $X_{2}=20 / 6 \mathrm{~nm}$.

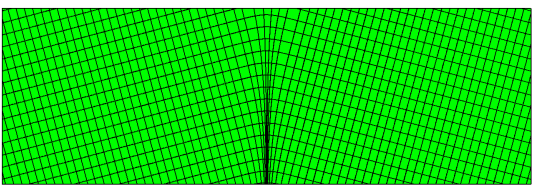

(a) $t=0 \mathrm{~s}$

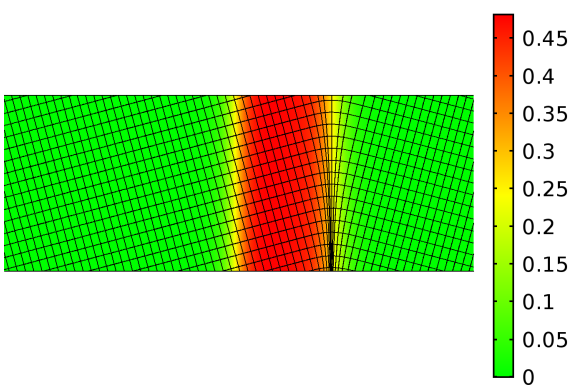

(b) $t=1 \times 10^{5} \mathrm{~s}$

Figure 9: Coupled grain boundary motion: Streamlines tangential to the vector fields $\boldsymbol{F}^{\mathrm{L}} \boldsymbol{e}^{1}$ and $\boldsymbol{F}^{\mathrm{L}} \boldsymbol{e}^{2}$ depicting the variations in the lattice, plotted in the reference configuration. The color density corresponds to the $1-2$ component of the plastic stain $\left(\boldsymbol{F}^{\mathrm{pT}} \boldsymbol{F}^{\mathrm{P}}-\boldsymbol{I}\right) / 2$.

The results of the simulation are shown in Figs. 7-9. The color plots of the initial and final GND density $G_{31}$ shown in Fig. 7 clearly demonstrate coupled motion. Fig. 8a shows the plots of $G_{31}$ and $G_{32}$ along the horizontal line $y=20 / 6 \mathrm{~nm}$ passing through the center of the domain. Compared to the plots at $t=0$, the dislocation density is more diffused at $t=1 \times 10^{5} \mathrm{~ns}$ which can be attributed to the stressed state of the material. The translation of the grain boundary during the coupled motion is more explicit in Fig. $8 \mathrm{~b}$ which shows the plots of the lattice orientation for the initial and final configurations. The lattice orientation is the angle corresponding to the unique rotation tensor $\boldsymbol{R}^{\mathrm{L}}$ that is obtained through the polar decomposition of $\boldsymbol{F}^{\mathrm{L}}$, i.e. $\boldsymbol{F}^{\mathrm{L}}=\boldsymbol{R}^{\mathrm{L}} \boldsymbol{U}^{\mathrm{L}}$. In order to visualize the changes in the lattice, we plot the streamlines of the vector fields $\boldsymbol{F}^{\mathrm{L}} \boldsymbol{e}^{1}$ and $\boldsymbol{F}^{\mathrm{L}} \boldsymbol{e}^{2}$, where $\boldsymbol{e}^{1}=(1,0)$ and $\boldsymbol{e}^{2}=(0,1)$ in Fig. 9. In addition, the color density of the $1-2$ component of plastic shear $\left(\boldsymbol{F}^{\mathrm{pT}} \boldsymbol{F}^{\mathrm{P}}-\boldsymbol{I}\right) / 2$, shown in Fig. 9, clearly demonstrates that the grain boundary plastically distorts the material as it sweeps through the material. The extent of coupling in a grain boundary motion is quantified using the coupling factor $\beta$ which is defined as the ratio of the distance covered by the grain boundary in the normal direction to that in the tangential direction. From (46), (49), (51) and (55), it is clear that for a coupled motion of a sharp-interface grain boundary, the coupling factor is equal to $2 \tan \left(\theta_{0} / 2\right)$, while it is zero for grain boundary sliding. In the simulation of coupled grain boundary motion, $\beta^{-1}$ is measured as the ratio of vertical displacement of the boundary $X_{1}=L$ and the distance traversed by the grain boundary measured using Fig. 8b. The plot of the inverse coupling factor versus time is shown in Fig. 10. Since the measurement of $\beta^{-1}$ is not a local measurement, it takes times to converge. From Fig. 10, it is clear that the converged value of $\beta^{-1}$ is below the theoretical value of 1.866. This can be attributed to the diffuse nature of the grain boundary which results in some sliding during the predominantly coupled grain boundary motion shown in Figs. 7-10.

Next, we simulate grain boundary sliding by replacing one of the slip system $(1,0)$ in the earlier simulation with $(0,1)$. The color plot of GND density $G_{31}$ in Fig. 11 shows a stationary grain boundary, clearly demonstrating grain boundary sliding. Fig. $11 \mathrm{~b}$ show the plots of $G_{31}$ and $G_{32}$ along the horizontal line $y=20 / 6 \mathrm{~nm}$ in the center of the domain for grain boundary sliding. From Fig. 11b, we note a small offset in the final position of the grain boundary relative to its initial position. This can be attributed to a small degree of coupling due to the diffuse nature of the grain boundary. 


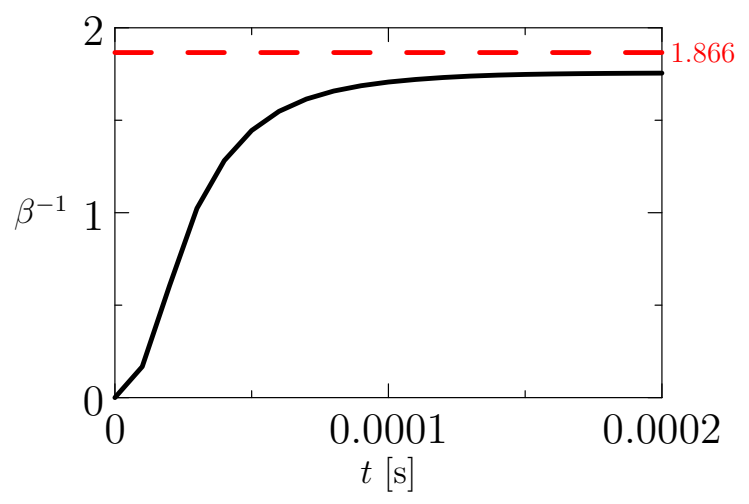

Figure 10: Coupled grain boundary motion: Plot showing the convergence of the inverse coupling factor $\beta^{-1}$ with respect to time. The value 1.866 is the theoretical $\beta^{-1}$ corresponding to the misorientation of $30^{\circ}$.
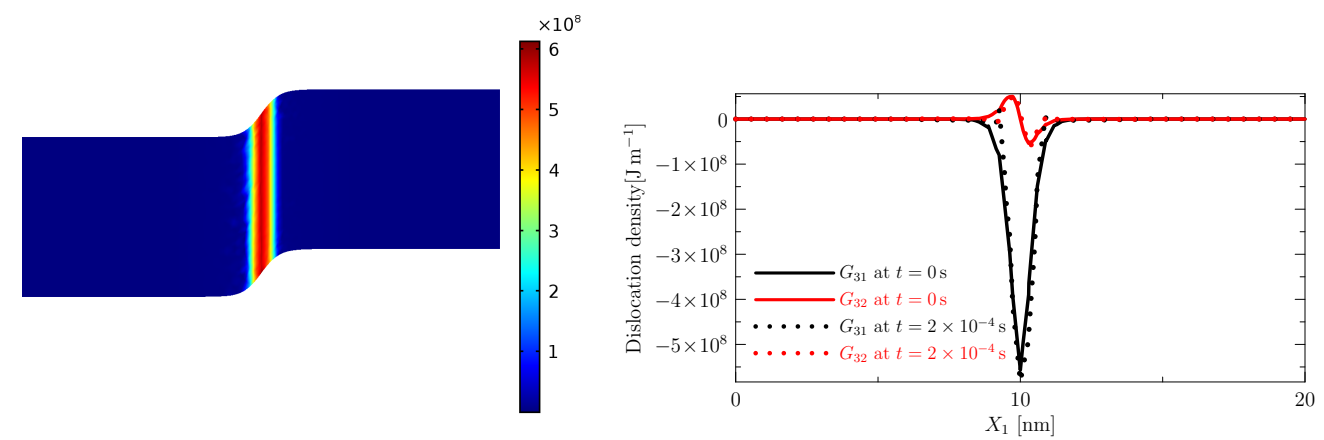

Figure 11: GB Sliding: a) Color plot of the norm of the GND density in the deformed configuration, and b) Plots of $G_{31}$ and $G_{32}$ as functions of the material coordinate $X_{1}$.

Summarizing the results of the analysis and simulations presented in this section, we note that grain boundary coupling and sliding are two independent mechanisms which can be activated depending on the choice of corresponding slip systems. Interestingly, the above discussion sheds light on an alternate kinematic mechanism for the translation of a flat grain boundary wherein $\boldsymbol{F}^{\mathrm{P}}$ remains a piecewise-constant rotation field, with the discontinuity translating with time, and $\boldsymbol{F}^{\mathrm{L}}=\left(\boldsymbol{F}^{\mathrm{P}}\right)^{\mathrm{T}}$, which implies $\boldsymbol{F}(\boldsymbol{X}, t)=\boldsymbol{I}$ for all time. In this mechanism, the grain boundary translates along its normal with no macroscopic deformation. Clearly, there is no driving force to activate this mechanism for a stressed/unstressed elastically isotropic bicrystal with a flat grain boundary. On the other hand, we postulate that for a stressed elastically anisotropic bicrystal with a flat grain boundary, a driving force exists which could activate this mechanism. In the next section, we show that the above mentioned mechanism is responsible for the shrinking of a circular grain, where the driving force originates from the nonzero curvature.

\subsection{Curvature-induced grain boundary motion and grain rotation}

In this section, with study grain boundary motion with curvature as the only driving force. Prior studies on the evolution of a circular grain have identified primarily three different kinds of grain evolution: 1) Grain rotation with no shrinkage, 2) grain shrinkage with no rotation, and 3) simultaneous grain rotation and shrinkage. In the latter, the interior grain can rotate either to increase or decrease the misorientation depending on whether the dislocations are conserved or not respectively. Recall from Section 7.2 that grain boundary coupling and sliding are defined for a flat grain boundary. Applying these definitions locally for a circular grain boundary, it can be easily shown that a coupled grain boundary motion involves the conservation of the dislocation content resulting in grain shrinkage and an increase in the misorientation. On the other hand, grain rotation with no shrinkage and a decreasing misorientation results in maximum rate at which dislocations are annihilated, and this corresponds to grain boundary sliding. Therefore, the rate of dislocation annihilation during grain shrinkage with no rotation lies in between that observed in coupling and sliding motions. 


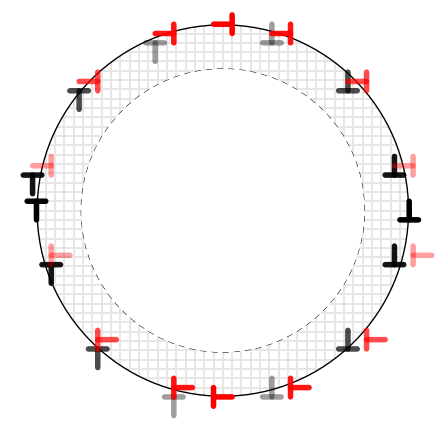

(a)

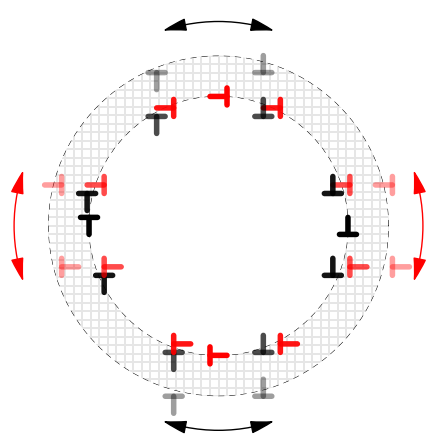

(b)

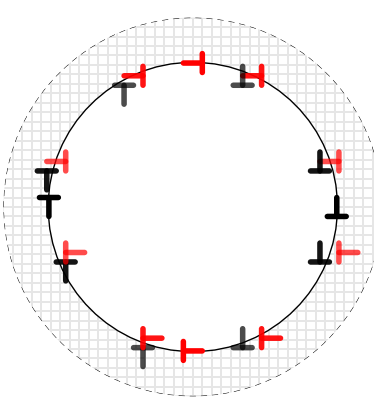

(c)

Figure 12: Mechanism for grain shrinkage with no rotation shown in the reference configuration, with the grid describing the slip planes. The circle drawn in solid line describes the position of the grain boundary. The GNDs on the grain boundary corresponding to the two slip systems are shown in red and black with the color intensity describing the magnitude of the dislocation density. Grain shrinkage occurs as the GNDs glide on their respect slip planes towards the interior of the grain. The four dislocations on the original grain boundary, as shown in (b), form pairs (marked by arrows) and move tangential to the grain boundary to annihilate, resulting in grain boundary shrinkage as shown in (c).

In this numerical study we perform two simulations to demonstrate (i) grain shrinkage with no rotation, and (ii) simultaneous grain shrinkage and rotation to decrease the misorientation angle. For simplicity, we consider a circular grain of initial radius $r_{0}$, with lattice orientation $\theta_{0} / 2$ embedded inside a medium with lattice orientation $-\theta_{0} / 2$. We begin by postulating that the mechanism involved in grain shrinkage is given by the following time-dependent plastic and elastic distortion fields:

$$
\begin{aligned}
& \boldsymbol{F}^{\mathrm{L}}\left(X_{1}, t\right)=\mathbb{1}_{\left(d(\boldsymbol{X})<r_{0}-t\right)} \boldsymbol{R}\left(\frac{\theta_{0}}{2}\right)+\mathbb{1}_{\left(d(\boldsymbol{X}) \geq r_{0}-t\right)} \boldsymbol{R}\left(-\frac{\theta_{0}}{2}\right), \\
& \boldsymbol{F}^{\mathrm{P}}\left(X_{1}, t\right)=\mathbb{1}_{\left(d(\boldsymbol{X})<r_{0}-t\right)} \boldsymbol{R}\left(-\frac{\theta_{0}}{2}\right)+\mathbb{1}_{\left(d(\boldsymbol{X}) \geq r_{0}-t\right)} \boldsymbol{R}\left(\frac{\theta_{0}}{2}\right),
\end{aligned}
$$

where $d(\boldsymbol{X}):=\sqrt{X_{1}^{2}+X_{2}^{2}}$. The equations in (60) result in $\boldsymbol{F}=\boldsymbol{I}$, which implies there is no macroscopic deformation. Additionally, the time-dependent GND tensor shown in Table 4 suggests the net dislocation content is directly proportional to the radius of the grain. As noted in Section 7.2, the evolution of GNDs is made possible by the availability of the slip planes. The expression for $\boldsymbol{L}^{\mathrm{P}}$ for the mechanism described in eq. (60) is shown in Table 4. Since the $1-2$ and $2-1$ components are the only non-zero components, the two slip systems given in Table 4 are sufficient for the evolution of GNDs. Moreover, it can also be shown that the same two slip systems also suffice for a diffuse grain boundary. Based on the mechanism given in (60), Fig. 12 depicts the motion of GNDs in a shrinking circular grain boundary, and shows how dislocations are transported and annihilated along the grain boundary.

We now simulate grain boundary shrinkage with no grain rotation in a square domain of size $40 \mathrm{~nm}$ with an embedded circular grain of radius $r_{0}=20 \mathrm{~nm}$ centered at the origin with radius $r_{0}=20 \mathrm{~nm}$. Based on the discussion following (60), we enable two slip systems with slip directions

$$
(1,0), \quad(0,1)
$$

The initial conditions for the simulation are

$$
\boldsymbol{u}(\boldsymbol{X}, 0)=\mathbf{0}, \quad \phi(\boldsymbol{X}, 0)=1, \quad \boldsymbol{F}^{\mathrm{P}}(\boldsymbol{X}, 0)=\boldsymbol{R}^{0 \mathrm{~T}}\left(\widetilde{\theta}\left(X_{1}\right)\right)
$$

where

$$
\widetilde{\theta}\left(X_{1}\right)=-\frac{\theta_{0}}{2}+\frac{\theta_{0}}{(1+\exp (-2.5(d(\boldsymbol{X})-20))},
$$

$\theta_{0}=60^{\circ}$, and $\boldsymbol{R}^{0}(\widetilde{\theta})$ is the rotation corresponding to $\widetilde{\theta}$. Dirichlet boundary conditions are enforced on the boundary of the square domain:

$$
\boldsymbol{u}(\partial \mathcal{B}, t)=\mathbf{0}, \quad \phi(\partial \mathcal{B}, t)=1, \quad v^{\alpha}(\partial \mathcal{B}, t)=0
$$




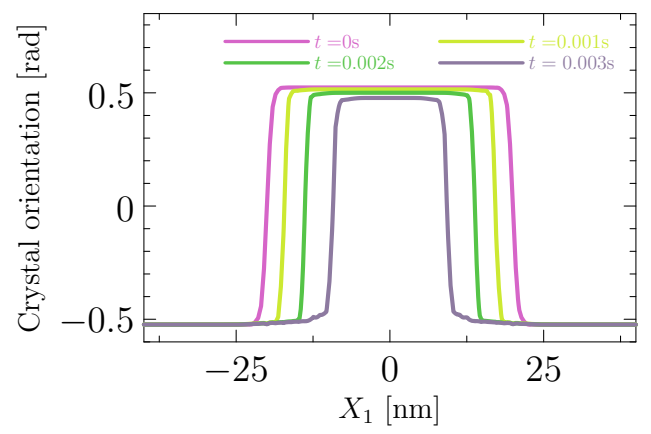

(a)
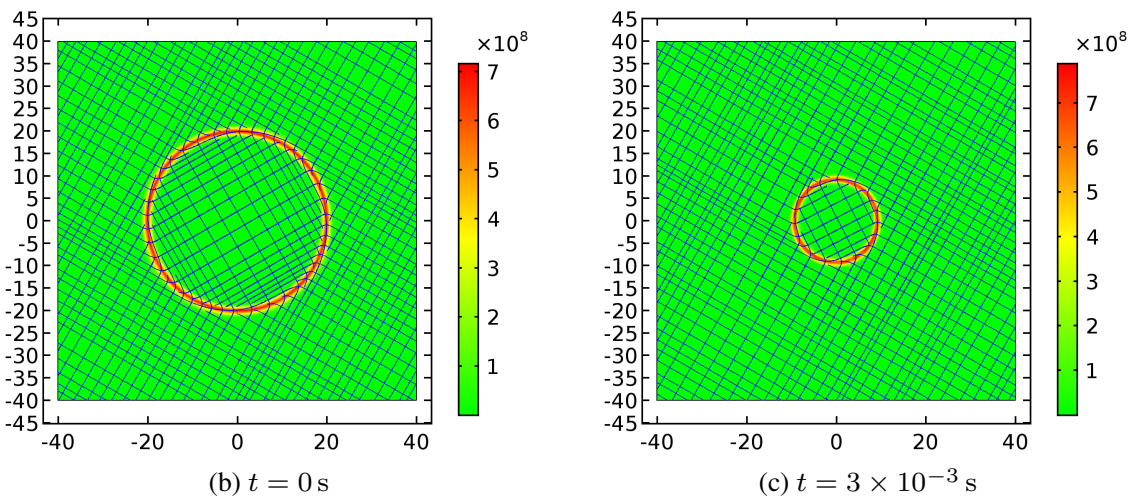

Figure 13: Grain shrinkage with no rotation: (a) Plots of the lattice orientation corresponding to the rotation $\boldsymbol{R}^{\mathrm{L}}$ obtained from the polar decomposition of $\boldsymbol{F}^{\mathrm{L}}$, along the horizontal central line $X_{2}=0$; (b), (c) Streamlines tangential to the vector fields $\boldsymbol{F}^{\mathrm{L}} \boldsymbol{e}^{1}$ and $\boldsymbol{F}^{\mathrm{L}} \boldsymbol{e}^{2}$ plotted in in the reference configuration depicting grain shrinkage with no rotation. The color density corresponds to the norm of GND density in units of $\mathrm{m}^{-1}$. The variations in the distances between parallel streamlines is an artefact of the algorithm used to plot them, and should not be interpreted as lattice stretches.

The functional form of mobilities $b^{\alpha}(\alpha=1,2)$, and $b^{\phi}$ chosen in Section 7.2 are left unchanged. The results of the simulations are shown in Fig. 13. Fig. 13a shows a plot of the lattice orientation $\theta^{\mathrm{L}}(\boldsymbol{X}, t)$ along the $X_{2}=0$, clearly demonstrating grain boundary shrinkage with negligible rotation. Figures $13 \mathrm{~b}$ and $13 \mathrm{c}$ display the structure of the grain at two different instants of time, 0 and $3 \times 10^{-3} \mathrm{~s}$. The color density in these figures corresponds to the norm of GND density in units of $\mathrm{m}^{-1}$.

Next, we simulate simultaneous grain shrinkage and rotation by activating all the four slip systems given in (41). The results of the simulation are shown in Fig. 14, where the plots clearly demonstrate simultaneous GB shrinkage and rotation. The two simulations described above highlight the relationship between slip systems and the mechanisms of grain boundary translation and rotation. Finally, Fig. 15 compares the norm of the plastic strain, i.e. $\left|\left(\boldsymbol{F}^{\mathrm{pT}} \boldsymbol{F}^{\mathrm{P}}-\boldsymbol{I}\right) / 2\right|$ for the two simulations described above. Fig. 15a shows negligible plastic strain for the case of grain shrinkage with no rotation, which is close to the zero plastic strain predicted by the mechanism given in (60). In addition, Fig. 15b confirms grain rotates without any plastic activity in its interior.

\subsection{Dynamic recovery}

In this section, we simulate the phenomenon of subgrain formation commonly referred to as dynamic recovery. During plastic deformation, the dislocation density in a material increases rapidly in stage II resulting in hardening. Following hardening, the existing dislocations consolidate forming dislocation cell walls. The cells are interpreted as "nucleated subgrains" because the dislocation density within each cell is smaller than in its boundary. Recovery precedes the stage of recrystallization which involves spontaneous growth of a cell at the expense of others into a dislocation free grain. Recovery during loading conditions is referred to as dynamic recovery.

Accordingly, the simulations are designed to deform a body and hold it in its deformed configuration enabling the buildup of dislocation content due to plastic distortion. The aim here is to study the long time evolution of the dislocation network to eventually nucleate new subgrains. The simulated domain is a rectangular single crystal of length $40 \mathrm{~nm}$ with an aspect ratio of $6: 1$ as shown in Fig. 16. The top surface is deformed gradually in the $X_{2}$ direction using a sinusoidal function with the maximum amplitude of $1 \mathrm{~nm}$ attained at $t=0.2 \mathrm{~s}$. The top surface is 


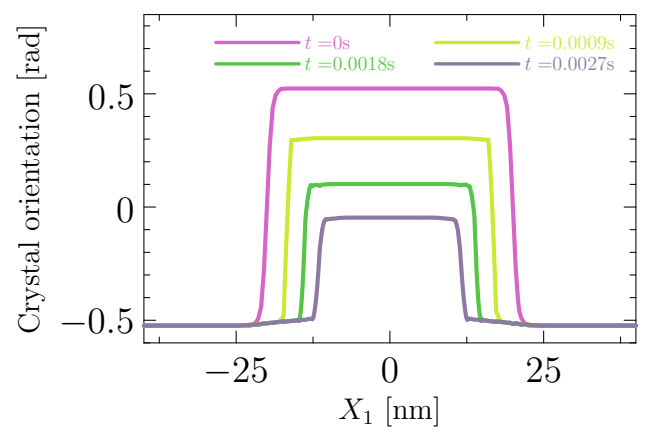

(a)
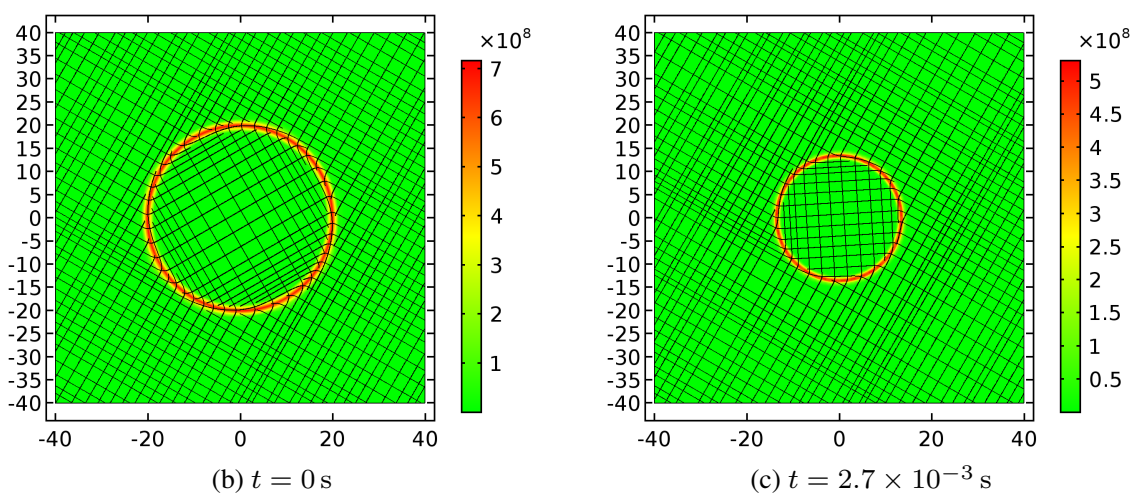

Figure 14: Grain shrinkage and rotation: (a) Plots of the lattice orientation corresponding to the rotation $\boldsymbol{R}^{\mathrm{L}}$ obtained from the polar decomposition of $\boldsymbol{F}^{\mathrm{L}}$, along the horizontal central line $X_{2}=0$; (b), (c) Streamlines tangential to the vector fields $\boldsymbol{F}^{\mathrm{L}} \boldsymbol{e}^{1}$ and $\boldsymbol{F}^{\mathrm{L}} \boldsymbol{e}^{2}$, plotted in the reference configuration, depicting grain shrinkage and rotation. The color density corresponds to the norm of GND density in units of $\mathrm{m}^{-1}$. The spacings between parallel streamlines is an artifact of the algorithm used to plot them, and should not be interpreted as lattice stretches.

then held in this position for the rest of the simulation. The remaining boundary conditions are all of the zero-flux kind. Unlike in previous simulations, by allowing zero-flux slip rate on the boundaries we have allowed for the buildup of a net non-zero dislocation content within the body. ${ }^{15}$ See Fig. 16 for the setup used in the simulation. The initial conditions are:

$$
\boldsymbol{u}(\boldsymbol{X}, 0)=\mathbf{0}, \quad \phi(\boldsymbol{X}, 0)=1, \quad \boldsymbol{F}^{\mathrm{P}}(\boldsymbol{X}, 0)=\boldsymbol{I}, \quad v^{\alpha}(\boldsymbol{X}, 0)=0
$$

In this case, the system is equipped with three slip systems with slip directions

$$
(1,0), \quad(1,1), \quad(-1,1)
$$

and inverse mobility $b^{1}$ is given by (59), while $b^{2}=b^{3}=10^{6} \times b^{1}$. In other words, dislocation activity in the first slip system is allowed while it is hindered in the second and third slip systems, which are included due to the diffuse nature of the model. The remaining material parameters for this simulation remain unchanged except for $\gamma$ which is set to $2000 \mathrm{~nm}$. This change is made to increase the tendency of bulk dislocations to form grain boundaries. ${ }^{16}$ The results of the simulation are shown in Figs. 17-18. Fig. 17 shows the color density plots of $|\boldsymbol{G}|$ for different times, plotted in the deformed configuration. The figure clearly demonstrates the buildup of bulk dislocations in the beginning of the simulation, which later agglomerate to form two grain boundaries. Fig. 18 shows plots of the lattice rotation and plastic rotation which are obtained from the polar decomposition of $\boldsymbol{F}^{\mathrm{L}}$ and $\boldsymbol{F}^{\mathrm{P}}$ respectively. As seen in Fig. 18a, the formation of grain boundaries leads to a discontinuity in lattice rotation, resulting in a decrease in the gradient of lattice rotation in the interior of the newly-formed grain. Fig. $18 \mathrm{~b}$ demonstrates the convergence of $\theta^{\mathrm{P}}$ to a step function conveying the formation of grain boundaries.

\footnotetext{
${ }^{15}$ On the other hand, if $v^{\alpha}=0$ on the boundary, then the net dislocation content in the body does not change, and it remains zero since we start with a perfect crystal.

${ }^{16}$ See Section 7.1 for the physical interpretation of $\gamma$ in the current model.
} 


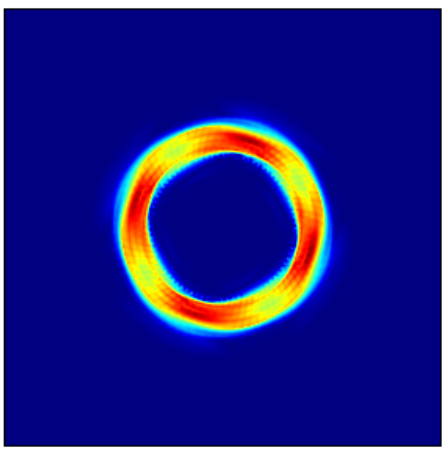

(a)

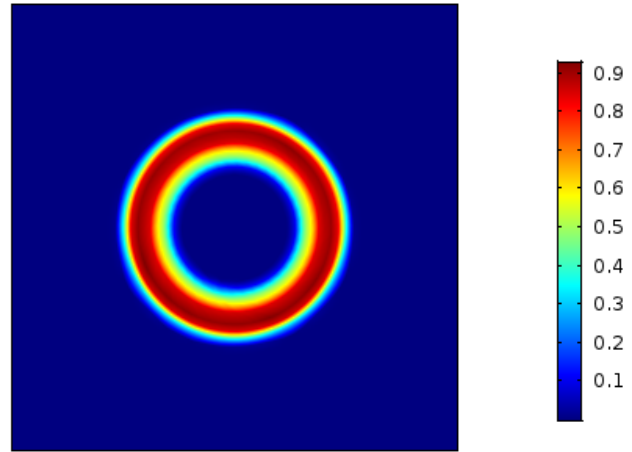

(b)

Figure 15: Comparison of color density plots of plastic strain $\left|\left(\boldsymbol{F}^{\mathrm{pT}} \boldsymbol{F}^{\mathrm{P}}-\boldsymbol{I}\right) / 2\right|$ for (a) grain shrinkage with no rotation, and (b) grain shrinkage with rotation, showing negligible plastic strain for pure shrinkage. Both plots confirm no dislocation activity in the grain interiors.

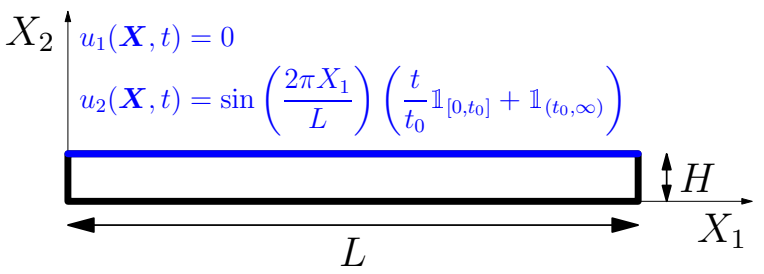

Figure 16: A schematic of a rectangular slab with $L=40 \mathrm{~nm}$ used to study dynamic recovery. The boundaries are subjected to zero-flux boundary condition in variables $\boldsymbol{u}, v^{\alpha}$ and $\phi$, except for a time-dependent Dirichlet boundary condition in the displacement variable on the top surface, with $t_{0}=0.2 \mathrm{~s}$.

\section{Discussion}

One of the most important issues in materials design is to understand the link between microstructure and properties $[47,48,49,50]$. This link often connects several orders of magnitude in space and time, which makes the formulation of physical models capable of spanning the relevant spatio-temporal gap an extremely challenging problem. While this topic has been attracting significant attention over the last two or so decades, our knowledge of the mechanisms that govern materials evolution under a number of important scenarios still presents many voids.

In terms of mechanical behavior, the study of polycrystal plasticity is one of the essential pillars supporting the development of new structural materials. Theory, modeling, and simulation has been consistently contributing to our understanding of the connection between microstructure and strength, fracture, or ductility. While tremendous progress has been made in the realm of 'static' properties, e.g. single dislocation properties [51], defect energetics and structure [52], grain boundary energies [53, 54], strengthening mechanisms [55, 56], etc., our understanding of the dynamic behavior of materials under stress at finite temperatures has been relatively lagging. This includes processes such as recovery, annealing, recrystallization, grain growth, etc., which are absolutely essential processes in materials synthesis and fabrication [57]. Under this same general category can be included processes such as superplasticity or Coble creep. This is the context within which we develop the present model: a thermodynamically-consistent approach that can simulate the dynamic evolution of polycrystals under the combined effects of temperature and mechanical deformation. The key points of our development are several. First, we utilize a special decomposition of the deformation gradient that allows us to define grain boundaries as a geometric link between a single crystal and a polycrystal. That is, rather than being ad-hoc structures introduced externally, grain boundaries appear naturally within our formulation, as a necessity to preserve the compatibility of the lattice in a multiple-grain configuration. Second, deformation and temperature-driven processes can be considered in unison in our model. This means that we can study mechanicallydriven processes, as well as thermal processes, in conjunction. This is of course one of the essential premises to model dynamic recovery and recrystallization during high-temperature deformation. Third, this dislocation-based definition of GBs allows for a seamless consideration of (dislocation-induced) bulk slip and grain boundary-mediated plasticity. This is guaranteed by the very definition of GBs within our formulation, which is done precisely in terms of a special 


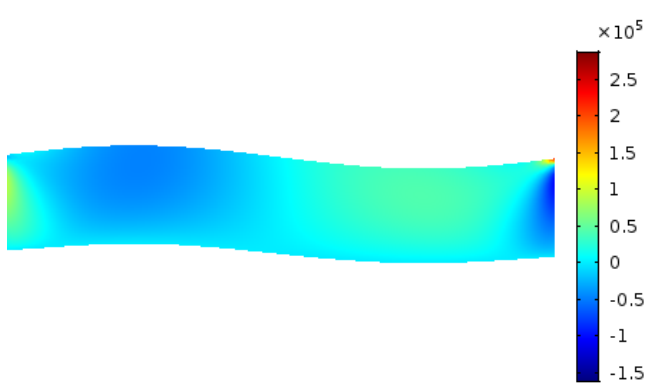

(a) $t=0.16 \mathrm{~s}$

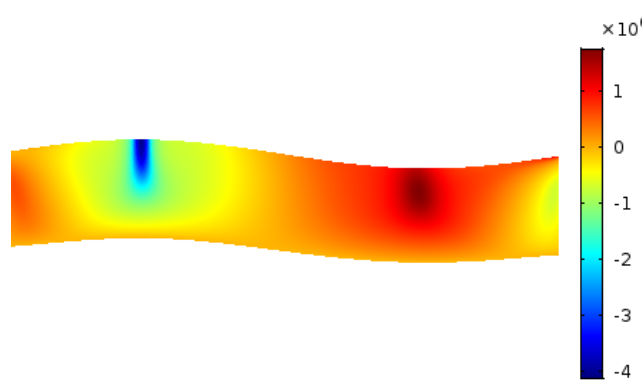

(c) $t=1.96 \mathrm{~s}$

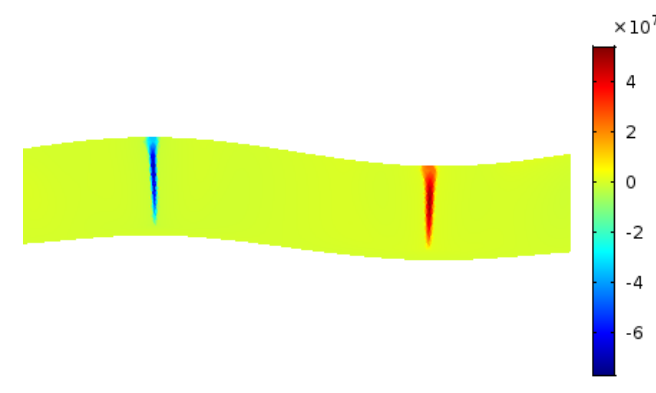

(e) $t=5 \mathrm{~s}$

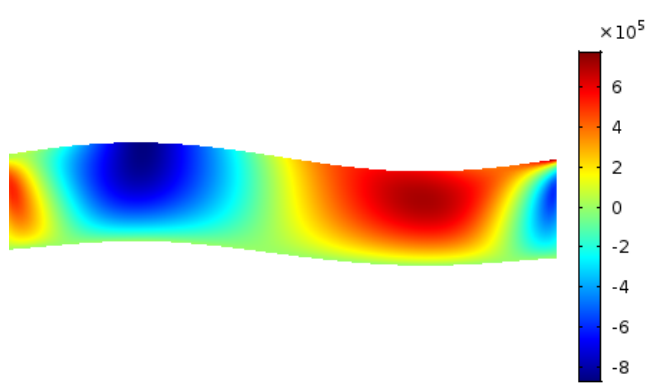

(b) $t=1.22 \mathrm{~s}$

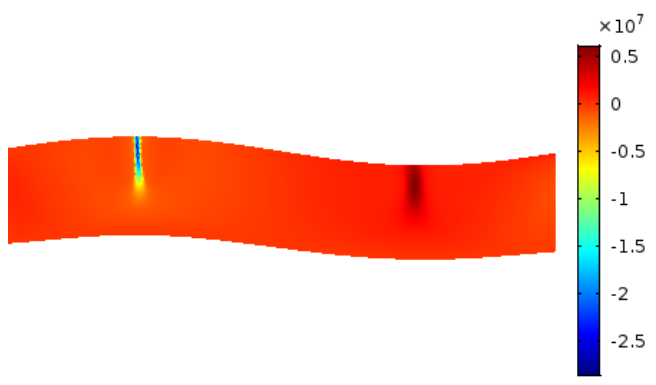

(d) $t=2.37 \mathrm{~s}$

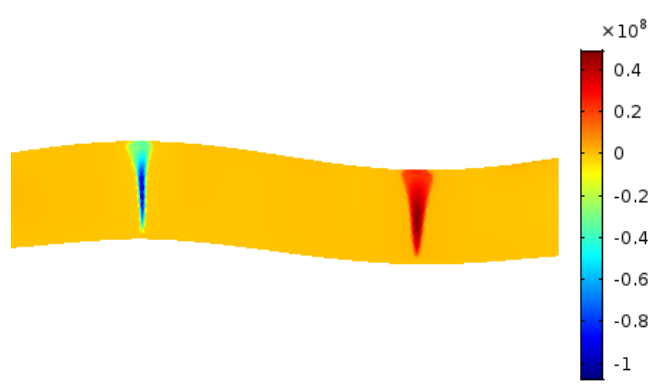

(f) $t=8.11 \mathrm{~s}$

Figure 17: Color density plots of $|\boldsymbol{G}|$ at different times, plotted in the deformed configuration, and in units of $\mathrm{m}^{-1}$.

class of dislocations. Lastly, the free energy expression employed here follows -at its core- a standard form commonly used in crystal plasticity, which is why our model is so easily integrable into existing crystal plasticity formulations.

These elements provide our model with a generality that we believe is quite unique. As discussed in Section 1 , the state of the art at present in the modeling of coupled bulk and GB-mediated plasticity involves evolving the GB microstructure and the dislocation network separately, and linking them externally via some penalty function. Since our model originates from a single free energy density, it truly permits simulating the co-evolution within the same framework of both sub-structures, enabling the consideration of dynamic processes under deformation. Specifically, we have looked at stress-driven processes and curvature-driven processes. From among the stress-driven transformations, we have studied the two mechanisms: shear-induced grain boundary motion and GB sliding. Their range of operation is thought to be clearly differentiated by temperature with coupled motion occurring primarily at low temperatures, and sliding at higher temperatures. Our model can yield the so-called coupling factor $\beta$, relating GB motion and grain translation, which makes it amenable to comparison to molecular dynamics simulations [46, 58, 59, 60, 61]. In fact, for a coupled GB motion, $\beta$ is know to be a geometric factor that depends only on the misorientation, and not on the inclination of the grain boundary. While various atomistic simulations have demonstrated coupled boundary motion in symmetric tilt GBs, the same is hard to reproduce for asymmetric tilt GBs [62]. However, it can be shown in a similar 

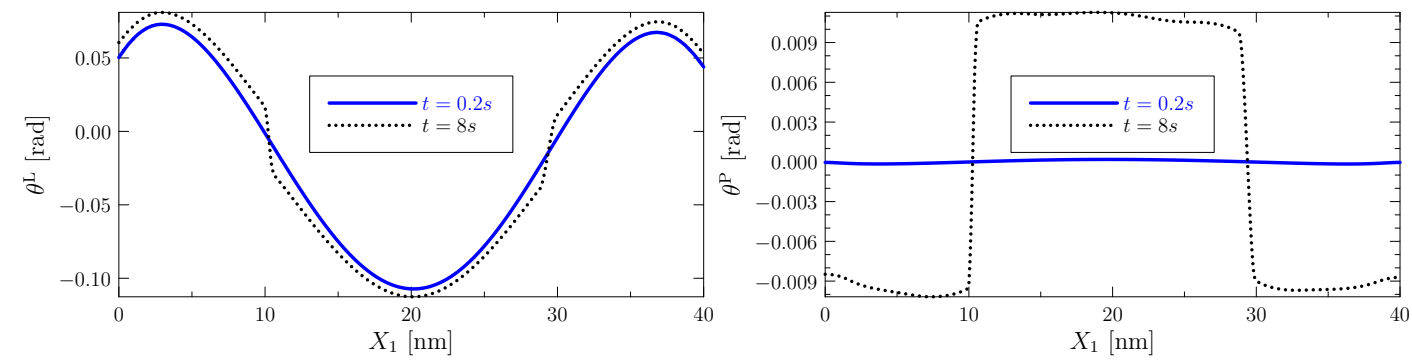

Figure 18: Plots of lattice rotation, and plastic rotation plotted as functions of the material coordinate $X_{1}$, along the line $X_{2}=20 / 12 \mathrm{~nm}$. $\theta^{\mathrm{L}}$ and $\theta^{\mathrm{P}}$ are angles corresponding to $\boldsymbol{R}^{\mathrm{L}}$ and $\boldsymbol{R}^{\mathrm{P}}$ in $S O(3)$ obtained from the polar decomposition of $\boldsymbol{F}^{\mathrm{L}}$ and $\boldsymbol{F}^{\mathrm{P}}$ respectively.

fashion as in Section 7.2 that normal stresses are needed to induce the necessary plastic distortion in asymmetric tilt boundaries. We conjecture that imposing the necessary stress boundary conditions predicted by the current framework in an MD simulation of an asymmetric tilt GB, would result in a coupled grain boundary motion. Sliding, for its part, occurs primarily at higher temperatures and low strain rates, and contributes for instance to creep, superplasticity, failure of ceramic materials at high temperature, etc [63, 64, 65].

Regarding curvature-driven processes, they appear to decrease the excess energy of a polycrystalline body ${ }^{17}$. From a fundamental point of view, grains can shrink to reduce the GB area while keeping the misorientation constant (grain shrinkage), or maintain their size while changing their misorientation to lower GB energy values (grain rotation) [66]. Evidently, both phenomena are most commonly found simultaneously during materials deformation, and both involve interactions between grain boundary dislocations, as we have shown here. All the processes mentioned above have been profusely investigated computationally in recent years [67, 6, 15, 14], and our approach draws in fact on knowledge acquired from these works.

A different issue is the phenomenon of dislocation subgrain formation, commonly found at the end of dynamic recovery at elevated stress and/or temperature. Such process is defined by the self-assembly of stored dislocations above a critical density into lower-energy dislocation wall configurations. These walls demarcate so-called subgrains, which have been theorized to be the precursors to kinetic hardening and recrystallization $[68,69,70]$. The driving force behind this fragmentation of the polycrystal into a collection of subgrains has long been unclear, although it is thought to be related to slip and hardening inhomogeneities across different crystal orientations in the grains. These inhomogeneities must preserve the compatibility of the deformation nonetheless, resulting in different grains suffering different lattice rotations, thus constituting subgrains. It is then that the dislocations stored at the boundaries of the regions change their character to GNDs, forming subgrain boundaries without long-range stresses. A recent study by Xia and El-Azab [71] has demonstrated the formation of subgrains using a mean field continuum dislocation dynamics model, with cross slip playing a pivotal role. However, it is important to note that the model presented by Xia and ElAzab [71] is a geometrically linear model, and the only driving force on dislocations is due to the resolved shear stress. On the other hand, our current geometrically non-linear kinematic framework clearly shows that one can construct a smooth dislocation density field (using arbitrary rotation fields) that results in zero stress. In such a scenario, in the presence of no resolved shear stress, there are no driving forces on the dislocations to form subgrains. This clearly highlights the importance of additional driving forces arising from higher-order stresses $\left(\xi^{\alpha}\right)$ that are responsible for subgrain formation. As shown in Section 7.4, we have induced the formation of dislocation walls by bending stresses, which has been suggested as the intrinsic cause behind the formation of dislocation cells and subgrains [70]. Our simulations show a very clean assemblage of dislocations into walls and the formation of misoriented grain boundaries. While we do not allege to solve but a small aspect of a complex and rich process, we believe that our model is capable of simulating the relevant mechanisms of polygonization, and we continue to further study this process.

The elementary GB phenomena discussed here have been simulated for model demonstration purposes. Ultimately, the goal is to combine all these processes under a single simulation scenario, to study phenomena such as dynamic recovery, dynamic recrystallization, polygonization, etc., and provide an avenue for validation and benchmarking. In addition, we intend to parameterize the approach using atomistic calculations, following recent trends in the community worldwide. This is the subject of ongoing studies by the authors for which significant efforts in numerical efficiency

${ }^{17}$ W.r.t. a single crystal, i.e., the product of the grain boundary area and the grain boundary energy 
and optimization of the implementation will be required. However, we are confident that our model will open up new opportunities to investigate the complex phenomena associated with polycrystal plasticity.

\section{Final summary}

As a final summary, the main features of the present work are listed below.

1. The framework used to construct the initial lattice strain-free polycrystal begins with a single crystal as the reference configuration. Using the decomposition $\boldsymbol{F}^{\mathrm{L}}=\boldsymbol{R}^{0}(\boldsymbol{X})$ and $\boldsymbol{F}^{\mathrm{P}}=\left(\boldsymbol{F}^{\mathrm{L}}\right)^{\mathrm{T}}$ of $\boldsymbol{F}(\boldsymbol{X}, 0) \equiv \boldsymbol{I}$, where $\boldsymbol{R}^{0}$ is a smoothened piecewise constant rotation field, we obtain a lattice strain-free diffuse-interface polycrystal. The misorientation between adjacent grains is a consequence of the presence of GNDs, described by the tensor $\boldsymbol{G}=\boldsymbol{F}^{\mathrm{P}} \operatorname{Curl} \boldsymbol{F}^{\mathrm{P}}$, concentrated at the grain boundaries. The framework described above is kinematically nonlinear and holds for arbitrary misorientations. Geometric nonlinearity plays a key role in obtaining lattice strain-free polycrystal because, in a linear theory, a non-zero dislocation density always results in a non-zero lattice strain.

2. The model includes grain boundary and bulk elastic energies. The bulk energy is the usual classical elastic energy which is a function of the lattice Lagrangian strain. The construction of grain boundaries using GNDs enables us to formulate the grain boundary energy as a function of $G$. The exact form of this function is the most non-trivial part of this paper. Inspired by the non-standard energy functional of the KWC model, which results in a singular diffusive equation for its order parameter $\theta$ that represents grain orientations, we construct the grain boundary energy by replacing $\nabla \theta$ appearing in the KWC energy density, with the GND density tensor $\boldsymbol{G}$.

3. The model has been applied to the following fundamental grain boundary processes (discussed in Section 7): planar grain boundary sliding and coupled motion, curvature-driven grain shrinkage, and curvature-driven grain rotation. As well, we have shown that our approach is capable of simulating the essential elements of the wellknown process of polygonization, i.e. the self-assembly of dislocations into cell walls that gives rise to the formation of subgrains. A length scale parameter $\gamma$ in the free energy functional describes the propensity of the dislocations to agglomerate to form grain boundaries. From a numerical viewpoint, increasing $\gamma$ increases the stiffness of the governing equations. Although the unified framework presented in this paper is dimensionindependent, all our simulations are implemented in 2-d. A three-dimensional implementation would differ only in the representation of the plastic rotation, used to solve the flow rule. In our recent work [34], where we introduced the abstract kinematic framework discussed in section 3, we have implemented a 3-d simulation of a polycrystal using an angle-axis representation of the plastic rotation. A similar strategy can be adopted for a 3-d simulation of the current model.

4. The approximation in the equivalence of $\boldsymbol{G}$ with $\nabla \theta$ results in residual lattice strains in the vicinity of the grain boundary of an unstressed bicrystal in steady state. While this artifact does not limit the model's capacity to simulate the various grain boundary-mediated plastic phenomena, we prove an interesting identity which states that the exact gradient of the lattice rotation can be additively decomposed into $G$ and a term that depends only on lattice stretch and its gradients. This lays the groundwork for a generalization of the current model, where $\nabla \theta$ of the KWC energy density is replaced by the exact lattice rotation gradient, thus resulting in a more accurate steady state solution with zero lattice strain in an unstressed bicrystal. Evidently, due to the presence of gradients in the lattice stretch, such a generalization results in a lattice strain gradient model which we will pursue in the near future.

\section{Acknowledgements}

Useful discussions with Timofey Frolov and Eliot Fried are acknowledged. NA and JM's work has been supported by the US Department of Energy's Office of Fusion Energy Sciences, grant DE-SC0012774:0001. Computer time allocations at UCLA's IDRE Hoffman2 supercomputer are acknowledged.

\section{Appendix A. Results on the 1-d KWC problem on $\Omega_{0}=[-L, L]$}

In this section, for the sake of completeness, we present a collection of results [35] for the solution to the 1-D KWC boundary value problem with boundary conditions

$$
\phi( \pm L)=1, \quad \theta( \pm L)= \pm \theta_{0} .
$$


As an ansatz, it is assumed that there exists a region in $\Omega_{0}$ given by $\Omega_{0}^{\mathrm{GB}}=(-l, l)$ where $\nabla \theta \neq \mathbf{0}$, while $\nabla \theta \equiv \mathbf{0}$ in the region $\left(\Omega_{0} \backslash \Omega_{0}^{\mathrm{GB}}\right)$, and $\phi$ and $\theta$ are symmetric and anti-symmetric respectively about the origin. Therefore, in the region $\Omega_{0} \backslash \Omega_{0}^{\mathrm{GB}}, \phi$ satisfies the equation

$$
\alpha^{2} \phi_{, X X}-f_{, \phi}=0 .
$$

Multiplying (A.2) by $\phi_{, X}$ and integrating, we obtain

$$
\frac{\alpha^{2}}{2} \phi_{, X}^{2}-f(\phi)=c, \quad\left(X \in \Omega_{0} \backslash \Omega_{0}^{\mathrm{GB}}\right)
$$

where $c$ is an integration constant. Integrating (A.3), we obtain the solution for $\phi$ outside $\Omega_{0}^{\mathrm{GB}}$ in its inversed form as

$$
L-X=\int_{\phi}^{1} \frac{\alpha}{\sqrt{2(f(\tilde{\phi})+c)}} d \tilde{\phi} . \quad\left(X \in \Omega_{0} \backslash \Omega_{0}^{\mathrm{GB}}\right)
$$

In the region $\Omega_{0}^{\mathrm{GB}}$, since $\theta_{, X} \neq 0$, we have $\theta,{ }_{X} /\left|\theta,{ }_{X}\right| \equiv 1$. Therefore, $\phi$ and $\theta$ satisfy the equations

$$
\left.\begin{array}{l}
\alpha^{2} \phi_{, X X}-f_{, \phi}-s g_{, \phi}\left|\theta_{, X}\right|=0, \\
\left(\epsilon^{2} \theta_{, X}+s g(\phi)\right)_{, X}=0 .
\end{array}\right\}\left(X \in \Omega_{0}^{\mathrm{GB}}\right)
$$

Therefore, the term $\left(\epsilon^{2} \theta_{, X}+s g(\phi)\right)$ is constant in $\Omega_{0}^{\mathrm{GB}}$. Since $\theta_{X}(X)=0$ at $X= \pm l$, it follows that

$$
\theta_{, X}=\frac{s\left(g\left(\phi_{2}\right)-g(\phi)\right)}{\epsilon^{2}}, \quad\left(X \in \Omega_{0}^{\mathrm{GB}}\right)
$$

where $\phi_{2}:=\phi( \pm l)$. Substituting (A.6) into (A.5), and multiplying (A.5) by $\phi, X$ results in

$$
\left(\frac{\alpha^{2}}{2} \phi, X-f(\phi)+\frac{s^{2}}{2 \epsilon^{2}}\left(g\left(\phi_{2}\right)-g(\phi)\right)^{2}\right), X=0 . \quad\left(X \in \Omega_{0}^{\mathrm{GB}}\right)
$$

Integrating (A.7), and noting from (A.3) that

$$
\frac{\alpha^{2}}{2} \phi_{, X}^{2}( \pm l)-f\left(\phi_{2}\right)=c
$$

it follows that

$$
\frac{\alpha^{2}}{2} \phi, X-f(\phi)+\frac{s^{2}}{2 \epsilon^{2}}\left(g\left(\phi_{2}\right)-g(\phi)\right)^{2}=c . \quad\left(X \in \Omega_{0}^{\mathrm{GB}}\right)
$$

Further integrating (A.9) results in

$$
X(\phi)=\int_{\phi_{1}}^{\phi} \frac{\alpha}{\sqrt{2\left(f(\tilde{\phi})+c-\left(\frac{s}{\epsilon}\right)^{2}\left(g\left(\phi_{2}\right)-g(\tilde{\phi})\right)^{2}\right.}} d \tilde{\phi}, \quad\left(\phi<\phi_{2}, X \in \Omega_{0}^{\mathrm{GB}}\right)
$$

where $\phi_{1}:=\phi(0)$. The solution for $\theta$ in $\Omega_{0}^{\mathrm{GB}}$ is obtained by integrating (A.6) resulting in

$$
\begin{aligned}
\theta(\phi) & =\frac{1}{\epsilon^{2}} \int_{\phi_{1}}^{\phi} \frac{s\left(g\left(\phi_{2}\right)-g(\tilde{\phi})\right)}{\phi, X} d \tilde{\phi}, \quad\left(\phi<\phi_{2}\right) \\
& =\frac{\alpha s}{\epsilon^{2}} \int_{\phi_{1}}^{\phi} \frac{g\left(\phi_{2}\right)-g(\tilde{\phi})}{\sqrt{2(f(\tilde{\phi})+c)-\left(\frac{s}{\epsilon}\right)^{2}\left(g\left(\phi_{2}\right)-g(\tilde{\phi})\right)^{2}}} d \tilde{\phi} \quad\left(\phi<\phi_{2}\right)
\end{aligned}
$$

where in the last equality we have used the expression for $\phi, X$ given in (A.9). Summarising the solution, (A.4) and (A.10) describe $\phi$ in the regions $\Omega_{0} \backslash \Omega_{0}^{\mathrm{GB}}$ and $\Omega_{0}^{\mathrm{GB}}$ respectively, while (A.11) describes $\theta$ in the region $\Omega_{0}^{\mathrm{GB}}$. The 


\begin{tabular}{|c|l|}
\hline$\epsilon^{2}$ & $2.1333 \times 10^{-4} \mathrm{fJ} \mathrm{nm}^{-1}$ \\
$\alpha^{2}$ & $5.3 \times 10^{-3} \mathrm{fJ} \mathrm{nm}^{-1}$ \\
$s$ & $0.0017 \mathrm{fJ} \mathrm{nm}^{-2}$ \\
$e$ & $0.0021 \mathrm{fJ} \mathrm{nm}^{-3}$ \\
$\gamma$ & $500 \mathrm{~nm}$ \\
$\theta_{0}$ & $30^{\circ}$ \\
$L$ & $10 \mathrm{~nm}$ \\
\hline
\end{tabular}

Table A.5: Parameters used in the implementation of the KWC model

solution is expressed in terms of constants $c, \phi_{1}$ and $\phi_{2}$ which are obtained implicitly from the relations

$$
\begin{aligned}
\theta_{0} & =\frac{\alpha s}{\epsilon^{2}} \int_{\phi_{1}}^{\phi_{2}} \frac{g\left(\phi_{2}\right)-g(\tilde{\phi})}{\sqrt{2(f(\tilde{\phi})+c)-\left(\frac{s}{\epsilon}\right)^{2}\left(g\left(\phi_{2}\right)-g(\tilde{\phi})\right)^{2}}} d \tilde{\phi} \\
c & =-f\left(\phi_{1}\right)+\frac{s^{2}}{2 \epsilon^{2}}\left(g\left(\phi_{2}\right)-g\left(\phi_{1}\right)\right)^{2}, \\
L & =\int_{\phi_{1}}^{\phi_{2}} \frac{\alpha}{\sqrt{2\left(f \left(\tilde{\phi}+c-\left(\frac{s}{\epsilon}\right)^{2}\left(g\left(\phi_{2}\right)-g(\tilde{\phi})\right)^{2}\right.\right.}} d \tilde{\phi}+\int_{\phi_{2}}^{1} \frac{\alpha}{\sqrt{2(f(\tilde{\phi})+c)}} d \tilde{\phi} .
\end{aligned}
$$

Finally, the grain boundary energy is defined as the minimum of the KWC free energy functional. It is instructive to observe the role of $\epsilon$ in the KWC energy functional. In the limit $\epsilon \rightarrow 0$, it is easy to see that $\phi_{2} \rightarrow \phi_{1}$, and $\theta$ converges to a step function with discontinuity at $X=0$. Therefore, the quadratic term in $\nabla \theta$ in the KWC energy functional serves as a regularization parameter.

\section{Motion by mean curvature and mobilities}

Lobkovsky and Warren [35] have shown that the sharp interface limit of equations (3a) and (3b) gives rise the grain rotation and grain boundary motion by curvature. In particular, the sharp interface limit was obtained by studying the scaled KWC Euler-Lagrange equations

$$
\begin{aligned}
\varepsilon b^{\phi} \dot{\phi} & =\varepsilon \alpha^{2} \triangle \phi-\frac{1}{\varepsilon} f^{\prime}(\phi)-s g_{\phi}|\nabla \theta|, \\
\varepsilon b^{\theta} \dot{\theta} & =\operatorname{Div}\left[\varepsilon \epsilon^{2} \nabla \theta+s g \frac{\nabla \theta}{|\nabla \theta|}\right],
\end{aligned}
$$

in the limit $\varepsilon \rightarrow 0$ using the method of matched asymptotics. The resulting sharp interface model is given by

$$
\mathfrak{v}=-\gamma \kappa M,
$$

where $\mathfrak{v}, \kappa$ and $M$ are the normal velocity, mean curvature, and mobility of the interface describing the grain boundary, and $\gamma$ is the grain boundary energy. The constants $M$ and $\gamma$ are given in terms of the solution to (3) described above for $L=\infty$ as

$$
M^{-1}=\int_{0}^{\infty}\left(b^{\phi} \phi,{ }_{X}^{2}+b^{\theta} \theta, \underset{X}{2}\right) d X
$$

and $\gamma$ is the corresponding evaluation of the KWC energy functional. Note that in the limit $\epsilon \rightarrow 0$, the mobility tends to zero. This implies, in addition to being a regularization parameter, $\epsilon$ plays an important role in rendering positive mobility to the phase field model.

\section{Numerical implementation of the KWC model in 1-d}

We now present details of the numerical implementation of the KWC model in 1-d. The simulation plots are presented in Section 2. The governing equations given in (5) are numerically solved on the domain $[-L, L]$, with $L=20 \mathrm{~nm}$, using the finite element method. The initial and boundary conditions used for the simulation are

$$
\begin{aligned}
\phi(X, 0) & =1, \quad \theta(X, 0)=-\frac{\theta_{0}}{2}+\frac{\theta_{0}}{(1+\exp (-2.5 X))}, \\
\phi(L, t)=\phi(-L, t) & =1, \quad \theta(L, t)=-\theta(-L, t)=\theta_{0} / 2,
\end{aligned}
$$


where $\theta_{0}$ denotes the jump in the lattice orientation across the grain boundary. The KWC parameters used for the simulation are listed in Table A.5. In addition, the inverse mobilities $b^{\phi}$ and $b^{\theta}$ are assumed to be constant, and equal to 1 (fJ ns) $/ \mathrm{nm}^{3}$. The variables $\theta$ and $\phi$ are interpolated using the Lagrange quadratic finite elements. Equations in (5) are solved using the MUMPS direct solver, and BDF (Backward Differential Formula) time stepping algorithm implemented in COMSOL 5.2. The system is evolved until a steady state is reached. Fig. 1a shows a comparison of the steady state solution obtained analytically and numerically. It is clear that $\nabla \theta$ in the numerical solution is not identically equal to zero. This is a result of simulating the approximate model governed by (5) as opposed to (3). It can the easily observed, although not shown here, that as $\gamma$ increases the two solutions converge. ${ }^{18}$

Next, we demonstrate grain shrinking and rotation of a circular grain of radius $20 \mathrm{~nm}$ with a misorientation of $60^{\circ}$ embedded inside a square domain of size $80 \mathrm{~nm}$. The initial and boundary conditions are given by

$$
\begin{aligned}
\theta(\Omega, 0)=-\frac{\theta_{0}}{2}+\frac{\theta_{0}}{1+\exp \left(-2.5\left(\sqrt{X_{1}^{2}+X_{2}^{2}}-20\right)\right)}, & \phi(\Omega, 0)=1, \\
\theta(\partial \Omega, t)=\theta(\partial \Omega, 0), & \phi(\partial \Omega, t)=1
\end{aligned}
$$

respectively. The system lower its free energy by a combination of grain shrinking and rotation. The two modes can be explored independently by appropriately choosing the mobilities. Grain rotation is a result of choosing constant mobilities, equal to 1 (fJ ns) $/ \mathrm{nm}^{3}$, while

$$
\begin{aligned}
& \left(b^{\phi}\right)^{-1}=1 \mathrm{~nm}^{3} /(\text { fJ ns }), \\
& \left(b^{\theta}\right)^{-1}=m_{\min }^{\theta}+\left(1-\phi^{3}\left(10-15 \phi+6 \phi^{2}\right)\right)\left(m_{\max }^{\theta}-m_{\min }^{\theta}\right),
\end{aligned}
$$

where $m_{\max }^{\theta}=1 \mathrm{~nm}^{3} /(\mathrm{fJ} \mathrm{ns})$ and $m_{\min }^{\theta}=1 \times 10^{-9} \mathrm{~nm}^{3} /(\mathrm{fJ} \mathrm{ns})$ results in pure shrink. The two modes are shown in Fig. 2.

\section{Appendix B. The Coleman-Noll procedure}

In this section, we use the Coleman-Noll procedure to arrive at a thermodynamically-consistent constitutive law for our model. As mentioned in Section 6, the free energy is assumed to be a function of $s=\left(T, \nabla T, \mathbb{E}^{\mathrm{L}}, \boldsymbol{G}, \phi, \dot{\phi}, \nabla \phi\right)$, $\boldsymbol{v}=\left(v^{1}, \ldots, v^{\alpha}\right)$ and $\nabla \boldsymbol{v}=\left(\nabla v^{1}, \ldots, \nabla v^{\alpha}\right)$, and the fields $\eta, \boldsymbol{q}, \boldsymbol{\xi}^{\alpha}, \pi, \Pi^{\alpha}$ and $\boldsymbol{S}$ are assumed to be functions of $s$, $\boldsymbol{v}, \nabla \boldsymbol{v}$ and $\boldsymbol{F}^{\mathrm{P}}$. Substituting these functional forms into the inequality in (29), we obtain

$$
\begin{aligned}
& \left(\psi,_{T}+\eta\right) \dot{T}+\psi, \nabla T \cdot \nabla \dot{T}+\left(\psi, \mathbb{E}^{\mathrm{L}}-\boldsymbol{S}\right) \cdot \dot{\mathbb{E}}^{\mathrm{L}}+\left(\psi,_{\phi}-\pi\right) \dot{\phi}+ \\
& (\psi, \nabla \phi-\mathfrak{p}) \cdot \nabla \dot{\phi}-\sum_{\alpha=1}^{A}\left(\Pi^{\alpha} v^{\alpha}+\tau^{\alpha} v^{\alpha}+\boldsymbol{\xi}^{\alpha} \cdot \nabla v^{\alpha}\right)+\psi,,_{\boldsymbol{G}} \dot{\boldsymbol{G}}+ \\
& \psi_{,_{\boldsymbol{v}}} \cdot \dot{\boldsymbol{v}}++\psi, \nabla \boldsymbol{v} \cdot \nabla \dot{\boldsymbol{v}}++\psi, \dot{\phi} \dot{\phi}+\frac{\boldsymbol{q} \cdot \nabla T}{T} \leq 0 .
\end{aligned}
$$

The above inequality must hold for all material points $\boldsymbol{X} \in \mathcal{B}^{0}$. Since $\nabla \dot{T}$ occurs in exactly one term in (B.1), and its coefficient is independent of $\nabla \dot{T}$, the inequality in (B.1) can be violated unless $\psi, \nabla \dot{T} \equiv 0$. By a similar argument, we have $\psi,_{\dot{\phi}} \equiv 0, \psi, \boldsymbol{v} \equiv \mathbf{0}$, and $\psi, \nabla \boldsymbol{v} \equiv \mathbf{0}$. Therefore, $\psi$ does not depend on $\nabla T, \dot{\phi}, \boldsymbol{v}$ and $\nabla \boldsymbol{v}$, resulting in a simplification of the inequality in (B.1) to

$$
\begin{aligned}
\left(\psi,,_{T}+\eta\right) \dot{T} & +\left(\psi,{ }_{\mathbb{E}^{\mathrm{L}}}-\boldsymbol{S}\right) \cdot \dot{\mathbb{E}}^{\mathrm{L}}+\left(\psi,_{\phi}-\pi\right) \dot{\phi}+(\psi, \nabla \phi-\mathfrak{p}) \cdot \nabla \dot{\phi}- \\
& \sum_{\alpha=1}^{A}\left(\Pi^{\alpha} v^{\alpha}+\boldsymbol{\xi}^{\alpha} \cdot \nabla v^{\alpha}+\tau^{\alpha} v^{\alpha}\right)+\psi,{ }_{\boldsymbol{G}} \dot{\boldsymbol{G}}+\frac{\boldsymbol{q} \cdot \nabla T}{T} \leq 0 .
\end{aligned}
$$

Using an argument similar to the one following (B.1), we conclude that $\eta, \boldsymbol{S}$ and $\mathfrak{p}$ are independent of $\nabla T, \boldsymbol{F}^{\mathrm{P}}, \boldsymbol{v}, \nabla \boldsymbol{v}$ and $\dot{\phi}$, and

$$
\begin{aligned}
\eta\left(T, \mathbb{E}^{\mathrm{L}}, \boldsymbol{G}, \phi, \nabla \phi\right) & =-\psi_{T}, \\
\boldsymbol{S}\left(T, \mathbb{E}^{\mathrm{L}}, \boldsymbol{G}, \phi, \nabla \phi\right) & =\psi,_{\mathbb{E}^{\mathrm{L}}}, \\
\mathfrak{p}\left(T, \mathbb{E}^{\mathrm{L}}, \boldsymbol{G}, \phi, \nabla \phi\right) & =\psi, \nabla \phi .
\end{aligned}
$$

\footnotetext{
${ }^{18}$ Of course, we cannot increase $\gamma$ indefinitely as this increases the stiffness of the resulting equations, thus resulting in higher computational cost.
} 
The term $\dot{G}$ appearing in (B.2) can be expressed in terms of the slip rates using the evolution equation (see Section 11.3 in Cermelli and Gurtin [72])

$$
\dot{\boldsymbol{G}}=\boldsymbol{L}^{\mathrm{P}} \boldsymbol{G}+\boldsymbol{G}\left(\boldsymbol{L}^{\mathrm{P}}\right)^{\mathrm{T}}+J^{\mathrm{P}} \sum_{\alpha=1}^{A}\left(\left(\boldsymbol{F}^{\mathrm{P}}\right)^{-T} \nabla v^{\alpha} \wedge \boldsymbol{m}^{\alpha}\right) \otimes \boldsymbol{s}^{\alpha},
$$

which implies

$$
\psi,_{\boldsymbol{G}} \cdot \dot{\boldsymbol{G}}=\sum_{\alpha=1}^{A}\left[\psi_{\boldsymbol{G}} \cdot\left(\mathbb{S}^{\alpha} \boldsymbol{G}+\boldsymbol{G}\left(\mathbb{S}^{\alpha}\right)^{\mathrm{T}}\right) v^{\alpha}+J^{\mathrm{P}}\left(\boldsymbol{F}^{\mathrm{P}}\right)^{-1}\left(\boldsymbol{m}^{\alpha} \wedge \psi,_{\boldsymbol{G}} \boldsymbol{s}^{\alpha}\right) \cdot \nabla v^{\alpha}\right] .
$$

Substituting (B.3) and (B.4) into the dissipation inequality (B.1), we obtain

$$
\begin{aligned}
\left(\psi, \phi_{\phi}-\pi\right) \dot{\phi}+\sum_{\alpha=1}^{A}\left[\left(\psi, \boldsymbol{G}:\left(\mathbb{S}^{\alpha} \boldsymbol{G}+\boldsymbol{G}\left(\mathbb{S}^{\alpha}\right)^{\mathrm{T}}\right)-\Pi^{\alpha}-\tau^{\alpha}\right) v^{\alpha}+\right. \\
\left.\left(J^{\mathrm{P}}\left(\boldsymbol{F}^{\mathrm{P}}\right)^{-1}\left(\boldsymbol{m}^{\alpha} \wedge \psi,_{\boldsymbol{G}} \boldsymbol{s}^{\alpha}\right)-\boldsymbol{\xi}^{\alpha}\right) \cdot \nabla v^{\alpha}\right]+\frac{\boldsymbol{q} \cdot \nabla T}{T} \leq 0 .
\end{aligned}
$$

We now assume that the microscopic stress $\boldsymbol{\xi}^{\alpha}$ is additively decomposed into an energetic part, which is independent of $\nabla \boldsymbol{v}$, and a dissipative part that depends on $\nabla \boldsymbol{v}$ :

$$
\boldsymbol{\xi}^{\alpha}=\boldsymbol{\xi}_{\mathrm{en}}^{\alpha}+\boldsymbol{\xi}_{\mathrm{d}}^{\alpha} .
$$

Substituting (B.6) into (B.5), and using the Coleman-Noll procedure, results in

$$
\boldsymbol{\xi}_{\text {en }}^{\alpha}=J^{\mathrm{P}}\left(\boldsymbol{F}^{\mathrm{P}}\right)^{-1}\left(\boldsymbol{m}^{\alpha} \wedge \psi, \boldsymbol{G} \boldsymbol{s}^{\alpha}\right),
$$

which implies $\boldsymbol{\xi}_{\mathrm{en}}^{\alpha}$ is the distributed Peach-Koehler force due to the pile up of dislocations, and $\boldsymbol{\xi}_{\mathrm{d}}^{\alpha}$ is the dissipative microstress conjugate to the gradient in slip rate. Substituting (B.6) and (B.7) into (B.5), the dissipation inequality reduces to

$$
\left(\psi,_{\phi}-\pi\right) \dot{\phi}+\sum_{\alpha=1}^{A}\left[\left(\psi, \boldsymbol{G}:\left(\mathbb{S}^{\alpha} \boldsymbol{G}+\boldsymbol{G}\left(\mathbb{S}^{\alpha}\right)^{\mathrm{T}}\right)-\Pi^{\alpha}-\tau^{\alpha}\right) v^{\alpha}-\boldsymbol{\xi}_{\mathrm{d}}^{\alpha} \cdot \nabla v^{\alpha}\right]+\frac{\boldsymbol{q} \cdot \nabla T}{T} \leq 0 .
$$

A solution to (B.8) is given by

$$
\begin{aligned}
\Pi^{\alpha} & =\psi,_{G}:\left(\mathbb{S}^{\alpha} \boldsymbol{G}+\boldsymbol{G} \mathbb{S}^{\alpha \mathrm{T}}\right)-\tau^{\alpha}+b^{\alpha}(s, \boldsymbol{v}, \nabla \boldsymbol{v}) v^{\alpha} \\
\boldsymbol{q} & =-\boldsymbol{K}(s, \boldsymbol{v}, \nabla \boldsymbol{v}) \nabla T \\
\pi & =\psi,_{\phi}+b^{\phi}(s, \boldsymbol{v}, \nabla \boldsymbol{v}) \dot{\phi} \\
\boldsymbol{\xi}_{\mathrm{d}}^{\alpha} & =B^{\alpha}(s, \boldsymbol{v}, \nabla \boldsymbol{v}) \nabla v^{\alpha}
\end{aligned}
$$

where $\boldsymbol{K}$ is the positive-definite thermal conductivity tensor, and the functions $b^{\alpha}, B^{\alpha}$ and $b^{\phi}$ are positive-valued inverse mobility functions associated with $v^{\alpha}, \nabla \boldsymbol{v}$ and $\dot{\phi}$ respectively.

\section{Appendix C. Gradient of lattice rotation}

In this section, we derive the following relation (see (39)) which expresses the gradient of lattice rotation in terms of the GND tensor, the stretch tensor $\boldsymbol{U}^{\mathrm{L}}$ obtained from the polar decomposition $\boldsymbol{F}^{\mathrm{L}}=\boldsymbol{R}^{\mathrm{L}} \boldsymbol{U}^{\mathrm{L}}$, and the gradient of $U^{\mathrm{L}}$ :

$$
\boldsymbol{R}^{\mathrm{LT}}\left(\operatorname{curl} \boldsymbol{R}^{\mathrm{LT}}\right)=\left(J^{\mathrm{L}}\right)^{-1}\left(\boldsymbol{U}^{\mathrm{L}} \boldsymbol{G} \boldsymbol{U}^{\mathrm{LT}}+\boldsymbol{U}^{\mathrm{L}} \overline{\operatorname{curl}} \boldsymbol{U}^{\mathrm{L}}\right),
$$

where curl and $\overline{\text { curl }}$ denote the curl operators with respect to the deformed and lattice configurations respectively.

We begin with an alternate representation of $\boldsymbol{G}$ in terms of $\boldsymbol{F}^{\mathrm{L}}$, given by

$$
\boldsymbol{G}=J^{\mathrm{L}} \boldsymbol{F}^{\mathrm{L}-1} \operatorname{curl} \boldsymbol{F}^{\mathrm{L}-1} .
$$


Substituting the polar decomposition of $\boldsymbol{F}^{\mathrm{L}}$ into (C.2), and simplifying the resulting expression using indicial notation, we obtain

$$
\begin{aligned}
G_{\bar{i} \bar{j}} & =J^{\mathrm{L}} F_{\bar{i} k}^{\mathrm{L}-1}\left[\operatorname{curl}\left(\boldsymbol{F}^{\mathrm{L}-1}\right)\right]_{k \bar{j}} \\
& =J^{\mathrm{L}} U_{\bar{i} \bar{k}}^{\mathrm{L}-1} R_{\bar{k} k}^{\mathrm{L}-1} \epsilon_{k r s} F_{\bar{j} s, r}^{\mathrm{L}-1} \\
& =J^{\mathrm{L}} U_{\bar{i} \bar{k}}^{\mathrm{L}-1} R_{\bar{k} k}^{\mathrm{L}-1} \epsilon_{k r s}\left[U_{\bar{j} \bar{l}, r}^{\mathrm{L}-1} R_{\bar{l} s}^{\mathrm{L}-1}+U_{\bar{j} \bar{l}}^{\mathrm{L}-1} R_{\bar{l} s, r}^{\mathrm{L}-1}\right],
\end{aligned}
$$

where letters in subscript appearing with a bar indicate components corresponding to the lattice configuration, while those in upper and lower case indicate components corresponding to the reference and deformed configurations respectively. Since $U_{\bar{j} \bar{l}}^{\mathrm{L}-1} U_{\bar{l} \bar{t}}^{\mathrm{L}}=\delta_{\bar{j} \bar{l}}$, its gradient with respect to the spatial coordinate is identically equal to zero. In other words,

$$
U_{\bar{j} \bar{l}, r}^{\mathrm{L}-1} U_{\bar{l} \bar{t}}^{\mathrm{L}}+U_{\bar{j} \bar{l}}^{\mathrm{L}-1} U_{\bar{l} \bar{t}, r}^{\mathrm{L}}=0 .
$$

This implies

$$
\begin{aligned}
U_{\bar{j} \bar{l}, r}^{\mathrm{L}-1} & =-U_{\bar{j} \bar{p}}^{\mathrm{L}-1} U_{\bar{t} \bar{l}}^{\mathrm{L}-1} U_{\bar{p} \bar{t}, r}^{\mathrm{L}} \\
& =-U_{\bar{j} \bar{p}}^{\mathrm{L}-1} U_{\bar{t} \bar{l}}^{\mathrm{L}-1} U_{\bar{p} \bar{t}, \bar{r}}^{\mathrm{L}} F_{\bar{r}, r}^{\mathrm{L}-1} \\
& =-U_{\bar{j} \bar{p}}^{\mathrm{L}-1} U_{\bar{t} \bar{l}}^{\mathrm{L}-1} U_{\bar{p} \bar{t}, \bar{r}}^{\mathrm{L}} U_{\bar{r}, \bar{s}}^{\mathrm{L}-1} R_{\bar{s} r}^{\mathrm{L}-1},
\end{aligned}
$$

where in the second equality we have expressed the gradient of the stretch tensor with respect to the coordinate in the lattice configuration. Substituting (C.5) into (C.3), we obtain

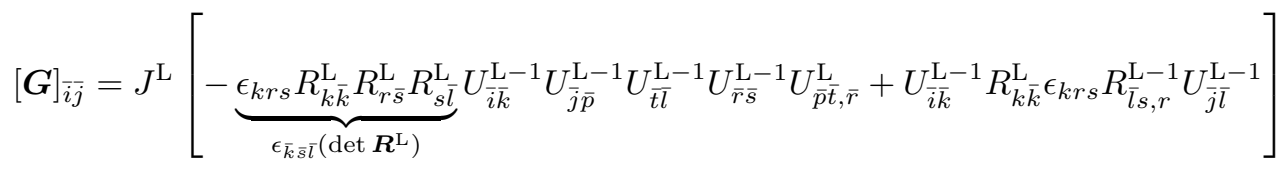

$$
\begin{aligned}
& =-J^{\mathrm{L}} \underbrace{\epsilon_{\bar{k} \bar{l}} U_{\overline{\bar{t}} \overline{\bar{l}}}^{\mathrm{L}-1} U_{\bar{r} \overline{\bar{s}}}^{\mathrm{L}-1} U_{\overline{\bar{l}}-1}^{\mathrm{L}-1}}_{\epsilon_{\bar{i} \bar{r} \bar{t}}\left(\operatorname{det} \boldsymbol{U}^{\mathrm{L}-\mathrm{T}}\right)} U_{\bar{j} \bar{p}}^{\mathrm{L}-1} U_{\bar{p} \bar{t}, \bar{r}}^{\mathrm{L}}+J^{\mathrm{L}}\left[\boldsymbol{U}^{\mathrm{L}-1} \boldsymbol{R}^{\mathrm{LT}}\left(\operatorname{curl} \boldsymbol{R}^{\mathrm{LT}}\right) \boldsymbol{U}^{\mathrm{L}-\mathrm{T}}\right]_{\bar{i} \bar{j}} \\
& =-\epsilon_{\bar{i} \bar{r} \bar{t}} U_{\bar{p} \bar{t}, \bar{r}}^{\mathrm{L}} U_{\bar{j} \bar{p}}^{\mathrm{L}-1}+J^{\mathrm{L}}\left[\boldsymbol{U}^{\mathrm{L}-1} \boldsymbol{R}^{\mathrm{LT}}\left(\operatorname{curl} \boldsymbol{R}^{\mathrm{LT}}\right) \boldsymbol{U}^{\mathrm{L}-\mathrm{T}}\right]_{\bar{i} \bar{j}} \\
& =\left[-\left(\overline{\operatorname{curl}} \boldsymbol{U}^{\mathrm{L}}\right) \boldsymbol{U}^{\mathrm{L}-\mathrm{T}}+J^{\mathrm{L}} \boldsymbol{F}^{\mathrm{L}-1}\left(\operatorname{curl} \boldsymbol{R}^{\mathrm{LT}}\right) \boldsymbol{U}^{\mathrm{L}-\mathrm{T}}\right]_{\bar{i} \bar{j}} .
\end{aligned}
$$

Expressing (C.6) in direct notation, we have

$$
\boldsymbol{G}=-\left(\overline{\operatorname{curl}} \boldsymbol{U}^{\mathrm{L}}\right) \boldsymbol{U}^{\mathrm{L}-\mathrm{T}}+J^{\mathrm{L}} \boldsymbol{F}^{\mathrm{L}-1}\left(\operatorname{curl} \boldsymbol{R}^{\mathrm{LT}}\right) \boldsymbol{U}^{\mathrm{L}-\mathrm{T}},
$$

which implies (C.1).

\section{References}

\section{References}

[1] P. Cotterill, P. R. Mould, Recrystallization and grain growth in metals, Krieger Pub Co, 1976.

[2] F. J. Humphreys, M. Hatherly, Recrystallization and related annealing phenomena, Elsevier, 2012.

[3] H. Van Swygenhoven, Grain boundaries and dislocations, Science 296 (2002) 66-67.

[4] L. Evers, W. Brekelmans, M. Geers, Scale dependent crystal plasticity framework with dislocation density and grain boundary effects, International Journal of solids and structures 41 (2004) 5209-5230.

[5] J. W. Cahn, Y. Mishin, A. Suzuki, Coupling grain boundary motion to shear deformation, Acta Materialia 54 (2006) 4953 - 4975. 
[6] M. Upmanyu, D. J. Srolovitz, A. Lobkovsky, J. A. Warren, W. Carter, Simultaneous grain boundary migration and grain rotation, Acta Materialia 54 (2006) 1707-1719.

[7] Y. Mishin, M. Asta, J. Li, Atomistic modeling of interfaces and their impact on microstructure and properties, Acta Materialia 58 (2010) 1117-1151.

[8] Z. Trautt, A. Adland, A. Karma, Y. Mishin, Coupled motion of asymmetrical tilt grain boundaries: Molecular dynamics and phase field crystal simulations, Acta Materialia 60 (2012) 6528-6546.

[9] D. Molodov, L. Barrales-Mora, J. Brandenburg, Grain boundary motion and grain rotation in aluminum bicrystals: recent experiments and simulations, in: IOP Conference Series: Materials Science and Engineering, volume 89, IOP Publishing, 2015, p. 012008.

[10] K. Elder, M. Katakowski, M. Haataja, M. Grant, Modeling elasticity in crystal growth, Physical review letters 88 (2002) 245701.

[11] K. Elder, M. Grant, Modeling elastic and plastic deformations in nonequilibrium processing using phase field crystals, Physical Review E 70 (2004) 051605.

[12] A. Yamanaka, K. McReynolds, P. W. Voorhees, Phase field crystal simulation of grain boundary motion, grain rotation and dislocation reactions in a bcc bicrystal, Acta Materialia 133 (2017) 160-171.

[13] V. Taupin, L. Capolungo, C. Fressengeas, M. Upadhyay, B. Beausir, A mesoscopic theory of dislocation and disclination fields for grain boundary-mediated crystal plasticity, International Journal of Solids and Structures 71 (2015) 277-290.

[14] R. Kobayashi, J. A. Warren, W. Craig Carter, A continuum model of grain boundaries, Physica D: Nonlinear Phenomena 140 (2000) 141-150.

[15] Kobayashi, Warren, Carter, Vector-valued phase field model for crystallization and grain boundary formation 119 (1998) 415-423.

[16] I. Steinbach, F. Pezzolla, A generalized field method for multiphase transformations using interface fields, Physica D: Nonlinear Phenomena 134 (1999) 385-393.

[17] C. K. III, L.-Q. Chen, Computer simulation of 3-d grain growth using a phase-field model, Acta Materialia 50 (2002) $3059-3075$.

[18] D. Raabe, Cellular automata in materials science with particular reference to recrystallization simulation, Annual review of materials research 32 (2002) 53-76.

[19] M. E. Gurtin, On the plasticity of single crystals: free energy, microforces, plastic-strain gradients, Journal of the Mechanics and Physics of Solids 48 (2000) 989-1036.

[20] M. E. Gurtin, A finite-deformation, gradient theory of single-crystal plasticity with free energy dependent on densities of geometrically necessary dislocations, International Journal of Plasticity 24 (2008) 702-725.

[21] A. Ma, F. Roters, D. Raabe, On the consideration of interactions between dislocations and grain boundaries in crystal plasticity finite element modeling-theory, experiments, and simulations, Acta Materialia 54 (2006) 2181-2194.

[22] P. Van Beers, G. McShane, V. Kouznetsova, M. Geers, Grain boundary interface mechanics in strain gradient crystal plasticity, Journal of the Mechanics and Physics of Solids 61 (2013) 2659-2679.

[23] S. Wulfinghoff, E. Bayerschen, T. Bhlke, A gradient plasticity grain boundary yield theory, International Journal of Plasticity 51 (2013) $33-46$.

[24] Y. Wei, L. Anand, Grain-boundary sliding and separation in polycrystalline metals: application to nanocrystalline fcc metals, Journal of the Mechanics and Physics of Solids 52 (2004) 2587-2616. 
[25] M. E. Gurtin, L. Anand, Nanocrystalline grain boundaries that slip and separate: A gradient theory that accounts for grain-boundary stress and conditions at a triple-junction, Journal of the Mechanics and Physics of Solids 56 (2008) 184-199.

[26] A. Basak, A. Gupta, Simultaneous grain boundary motion, grain rotation, and sliding in a tricrystal, Mechanics of Materials 90 (2015) 229-242.

[27] T. Frolov, Y. Mishin, Thermodynamics of coherent interfaces under mechanical stresses. i. theory, Physical Review B 85 (2012) 224106.

[28] D. Raabe, R. C. Becker, Coupling of a crystal plasticity finite-element model with a probabilistic cellular automaton for simulating primary static recrystallization in aluminium, Modelling and Simulation in Materials Science and Engineering 8 (2000) 445.

[29] G. Abrivard, E. P. Busso, S. Forest, B. Appolaire, Phase field modelling of grain boundary motion driven by curvature and stored energy gradients. part ii: application to recrystallisation, Philosophical magazine 92 (2012) $3643-3664$.

[30] G. Abrivard, E. P. Busso, S. Forest, B. Appolaire, Phase field modelling of grain boundary motion driven by curvature and stored energy gradients. part i: theory and numerical implementation, Philosophical magazine 92 (2012) 3618-3642.

[31] M. Bernacki, R. E. Logé, T. Coupez, Level set framework for the finite-element modelling of recrystallization and grain growth in polycrystalline materials, Scripta Materialia 64 (2011) 525-528.

[32] T. Takaki, T. Hirouchi, Y. Hisakuni, A. Yamanaka, Y. Tomita, Multi-phase-field model to simulate microstructure evolutions during dynamic recrystallization, Materials transactions 49 (2008) 2559-2565.

[33] E. Popova, Y. Staraselski, A. Brahme, R. Mishra, K. Inal, Coupled crystal plasticity probabilistic cellular automata approach to model dynamic recrystallization in magnesium alloys, International Journal of Plasticity 66 (2015) $85-102$.

[34] N. C. Admal, G. Po, J. Marian, Diffuse-interface polycrystal plasticity: expressing grain boundaries as geometrically necessary dislocations, Materials Theory 1 (2017) 6.

[35] A. Lobkovsky, J. Warren, Sharp interface limit of a phase-field model of crystal grains, Physical Review E 63 (2001) 051605.

[36] R. Kobayashi, Y. Giga, Equations with singular diffusivity, Journal of Statistical Physics 95 (1999) 1187-1220.

[37] E. H. Lee, Elastic-plastic deformation at finite strains, ASME, 1969.

[38] E. Kröner, Allgemeine kontinuumstheorie der versetzungen und eigenspannungen, Archive for Rational Mechanics and Analysis 4 (1959) 273-334.

[39] C. Reina, S. Conti, Kinematic description of crystal plasticity in the finite kinematic framework: a micromechanical understanding of $\mathrm{f}=\mathrm{f}$ e $\mathrm{f} \mathrm{p}$, Journal of the Mechanics and Physics of Solids 67 (2014) 40-61.

[40] A. Acharya, A counterpoint to cermelli and gurtins criteria for choosing the correctgeometric dislocation tensor in finite plasticity, in: IUTAM Symposium on theoretical, computational and modelling aspects of inelastic media, Springer, 2008, pp. 99-105.

[41] V. L. Berdichevsky, Continuum theory of dislocations revisited, Continuum Mechanics and Thermodynamics 18 (2006) 195-222.

[42] P. G. Ciarlet, G. Geymonat, Sur les lois de comportement en élasticité non linéaire compressible, CR Acad. Sci. Paris Sér. II 295 (1982) 423-426.

[43] J. Nye, Some geometrical relations in dislocated crystals, Acta metallurgica 1 (1953) 153-162.

[44] E. Kröner, et al., Continuum theory of defects, Physics of defects 35 (1981) 217-315. 
[45] W. T. Read, W. Shockley, Dislocation models of crystal grain boundaries, Physical review 78 (1950) 275.

[46] J. W. Cahn, Y. Mishin, A. Suzuki, Coupling grain boundary motion to shear deformation, Acta materialia 54 (2006) 4953-4975.

[47] M. Kumar, A. J. Schwartz, W. E. King, Microstructural evolution during grain boundary engineering of low to medium stacking fault energy fcc materials, Acta materialia 50 (2002) 2599-2612.

[48] D. L. McDowell, H. J. Choi, J. Panchal, R. Austin, J. Allen, F. Mistree, Plasticity-related microstructure-property relations for materials design, in: Key Engineering Materials, volume 340, Trans Tech Publ, 2007, pp. 21-30.

[49] D. L. McDowell, G. Olson, Concurrent design of hierarchical materials and structures, in: Scientific Modeling and Simulations, Springer, 2008, pp. 207-240.

[50] D. L. McDowell, A perspective on trends in multiscale plasticity, International Journal of Plasticity 26 (2010) 1280 - 1309. Special Issue In Honor of David L. McDowell.

[51] V. Bulatov, W. Cai, Computer simulations of dislocations, volume 3, Oxford University Press on Demand, 2006.

[52] P. Gehlen, Interatomic potentials and simulation of lattice defects, Springer Science \& Business Media, 2012.

[53] O. Shenderova, D. Brenner, A. Nazarov, A. Romanov, L. Yang, Multiscale modeling approach for calculating grain-boundary energies from first principles, Physical Review B 57 (1998) R3181.

[54] M. Tschopp, D. McDowell, Dislocation nucleation in 3 asymmetric tilt grain boundaries, International Journal of Plasticity 24 (2008) $191-217$.

[55] Y. N. Osetsky, D. J. Bacon, An atomic-level model for studying the dynamics of edge dislocations in metals, Modelling and simulation in materials science and engineering 11 (2003) 427.

[56] P. Erhart, J. Marian, B. Sadigh, Thermodynamic and mechanical properties of copper precipitates in $\alpha$-iron from atomistic simulations, Physical Review B 88 (2013) 024116.

[57] C. C. Tasan, M. Diehl, D. Yan, M. Bechtold, F. Roters, L. Schemmann, C. Zheng, N. Peranio, D. Ponge, M. Koyama, et al., An overview of dual-phase steels: advances in microstructure-oriented processing and micromechanically guided design, Annual Review of Materials Research 45 (2015) 391-431.

[58] J. W. Cahn, Y. Mishin, A. Suzuki, Duality of dislocation content of grain boundaries, Philosophical Magazine 86 (2006) 3965-3980.

[59] A. Suzuki, Y. M. Mishin, Atomic mechanisms of grain boundary motion, in: Materials Science Forum, volume 502, Trans Tech Publ, 2005, pp. 157-162.

[60] V. Ivanov, Y. Mishin, Dynamics of grain boundary motion coupled to shear deformation: An analytical model and its verification by molecular dynamics, Physical Review B 78 (2008) 064106.

[61] Z. Trautt, Y. Mishin, Grain boundary migration and grain rotation studied by molecular dynamics, Acta Materialia 60 (2012) 2407-2424.

[62] Z. Trautt, A. Adland, A. Karma, Y. Mishin, Coupled motion of asymmetrical tilt grain boundaries: Molecular dynamics and phase field crystal simulations, Acta Materialia 60 (2012) 6528-6546.

[63] R. Raj, M. Ashby, On grain boundary sliding and diffusional creep, Metallurgical and Materials Transactions B 2 (1971) 1113-1127.

[64] R. Gifkins, Grain-boundary sliding and its accommodation during creep and superplasticity, Metallurgical and Materials Transactions A 7 (1976) 1225-1232.

[65] H. Van Swygenhoven, P. Derlet, Grain-boundary sliding in nanocrystalline fcc metals, Physical Review B 64 (2001) 224105. 
[66] L. Margulies, G. Winther, H. Poulsen, In situ measurement of grain rotation during deformation of polycrystals, Science 291 (2001) 2392-2394.

[67] G. Gottstein, L. S. Shvindlerman, Grain boundary migration in metals: thermodynamics, kinetics, applications, CRC press, 2009.

[68] H. Mughrabi, Dislocation wall and cell structures and long-range internal stresses in deformed metal crystals, Acta metallurgica 31 (1983) 1367-1379.

[69] B. Bay, N. Hansen, D. Hughes, D. Kuhlmann-Wilsdorf, Overview no. 96 evolution of fec deformation structures in polyslip, Acta metallurgica et materialia 40 (1992) 205-219.

[70] R. Sedláček, W. Blum, J. Kratochvil, S. Forest, Subgrain formation during deformation: physical origin and consequences, Metallurgical and Materials Transactions A 33 (2002) 319-327.

[71] S. Xia, A. El-Azab, Computational modelling of mesoscale dislocation patterning and plastic deformation of single crystals, Modelling and Simulation in Materials Science and Engineering 23 (2015) 055009.

[72] P. Cermelli, M. E. Gurtin, Geometrically necessary dislocations in viscoplastic single crystals and bicrystals undergoing small deformations, International Journal of Solids and Structures 39 (2002) 6281-6309. 